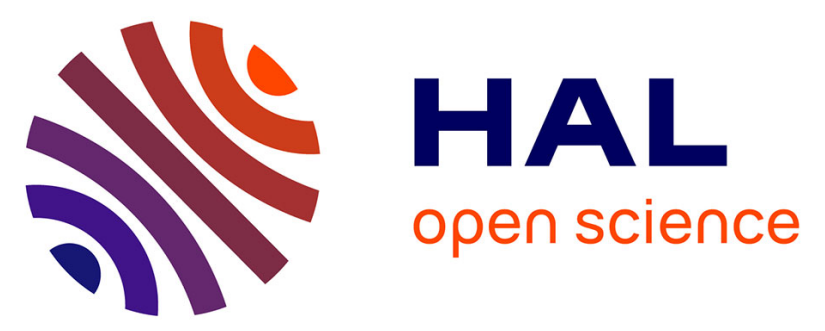

\title{
Instabilities in a cylindrical cavity heated from below with a free surface. I. Effect of Biot and Marangoni numbers.
}

\author{
Ridha Touihri, Anas El Gallaf, Daniel Henry, Hamda Ben Hadid
}

\section{To cite this version:}

Ridha Touihri, Anas El Gallaf, Daniel Henry, Hamda Ben Hadid. Instabilities in a cylindrical cavity heated from below with a free surface. I. Effect of Biot and Marangoni numbers.. Physical Review E: Statistical, Nonlinear, and Soft Matter Physics, 2011, 84, pp.056302. 10.1103/PhysRevE.84.056302 . hal-00691095

\section{HAL Id: hal-00691095 \\ https://hal.science/hal-00691095}

Submitted on 11 Apr 2016

HAL is a multi-disciplinary open access archive for the deposit and dissemination of scientific research documents, whether they are published or not. The documents may come from teaching and research institutions in France or abroad, or from public or private research centers.
L'archive ouverte pluridisciplinaire HAL, est destinée au dépôt et à la diffusion de documents scientifiques de niveau recherche, publiés ou non, émanant des établissements d'enseignement et de recherche français ou étrangers, des laboratoires publics ou privés. 


\title{
Instabilities in a cylindrical cavity heated from below with a free surface. I. Effect of Biot and Marangoni numbers
}

\author{
R. Touihri, ${ }^{1}$ A. El Gallaf, ${ }^{2}$ D. Henry, ${ }^{2}$ and H. Ben Hadid ${ }^{2}$ \\ ${ }^{1}$ LAMSIN, ENIT, Campus Universitaire, Le Belvédère, BP 37, TN-1002 Tunis, Tunisia \\ ${ }^{2}$ Laboratoire de Mécanique des Fluides et d'Acoustique, CNRS/Université de Lyon, Ecole Centrale de Lyon/Université Lyon 1/INSA de Lyon, \\ ECL, 36 Avenue Guy de Collongue, FR-69134 Ecully Cedex, France
}

(Received 23 December 2010; published 8 November 2011)

\begin{abstract}
Convective instabilities in a cylindrical cavity heated from below, with a free surface at the top, are numerically investigated using a spectral-element code. Both buoyancy and surface tension forces are taken into account, and heat exchange is considered at the upper surface. This configuration corresponds to the Bénard-Marangoni situation. The primary thresholds associated with azimuthal eigenmodes and corresponding to the onset of convection are first given as a function of the aspect ratio of the cavity $A$ (radius/height), the Biot number Bi, and the Marangoni number Ma. Particular attention is paid to the influence of the Biot and Marangoni numbers: a stabilizing surface tension effect $(\mathrm{Ma}>0)$ induces an increase of the primary thresholds, which is magnified for small values of $\mathrm{Bi}$, but may also change the flow structure by creating counter-rotating rolls near the free surface. The nonlinear evolution of the convection beyond its onset is given through bifurcation diagrams for $A=1.5$. Two different branches of axisymmetric solutions, either with upflow or downflow at the center, emerge at the onset. The destabilization of these solutions and the further dynamical evolution of the flow has been highlighted for widely varying Biot numbers.
\end{abstract}

DOI: 10.1103/PhysRevE.84.056302

PACS number(s): 47.20.Bp

\section{INTRODUCTION}

The onset of thermoconvective instabilities in a fluid layer heated from below with a free surface at the top is a classical problem in fluid mechanics since the seminal experiment of Bénard [1]. This problem has been much studied to elucidate pattern formation (for example, see the book of Koschmieder [2]). In this problem, it is well known that convection sets in when the temperature difference becomes larger than a certain critical value that depends on the shape of the container and its aspect ratio and on possible imposed constraints. Two different effects can be responsible for this instability. The first effect is buoyancy and is connected to density variations induced by temperature in the gravity field. The second effect is related to the capillary forces appearing at the free surface and varying with the temperature and is known as the Marangoni effect. The onset of convection due to buoyancy was first studied theoretically by Rayleigh [3] and assumed to be at the origin of the convective cells observed experimentally by Bénard [1]. Such a problem is referred to as the Rayleigh-Bénard problem. The onset of convection due to the capillary forces, which was in fact a better explanation of Bénard's observations, was studied later, in 1958, by Pearson [4]. This problem is often called the Marangoni problem. The situation wherein both effects are combined, which gives rise to the so-called Bénard-Marangoni instability, was then considered by Nield [5] in 1964.

Relatively few experimental studies have been devoted to Bénard-Marangoni convection in small aspect ratio containers. An important work is that of Koschmieder and Prahl [6], who considered small square and circular containers and studied the effect of the shape and aspect ratio of the container on the thresholds and on the structure of the emerging flow. Johnson and Narayanan [7] made experiments for circular containers with aspect ratios 1.5 and 2.5 , and showed the possibility of oscillatory motions due to the switching between two flow patterns. More recently, Cerisier et al. [8] and Rahal et al. [9] investigated the effect of the Prandtl and Biot numbers on the pattern selection and interface deformation.

In contrast, following the work of Pearson, many theoretical studies were devoted to this problem assuming horizontally infinite layers. Different stability approaches were used and the physical model was refined (see the references in [10]). Rosenblat et al. $[11,12]$ were the first to consider the problem of thermocapillary convection in finite boxes. They performed a linear and weakly nonlinear study for cylindrical and rectangular containers with free-slip sidewalls. This assumption of free-slip sidewalls was also later used by Dauby et al. [13], who analyzed the onset of steady convection with hexagonal structure. Linear and nonlinear studies of surface-tensiondriven convection for a container with rigid sidewalls were performed by Dijkstra either in two-dimensional [14] or in three-dimensional [15-17] situations. His results are in good agreement with experiments in small boxes. Dauby et al. [18] solved the same problem in three dimensions for containers with horizontal aspect ratios varying independently from 1 to 9. Their results obtained in the weakly nonlinear regime are in good agreement with the experimental results of Koschmieder and Prahl [6].

For the cylindrical geometry considered here, the problem has been studied in several papers. Vrentas et al. [19] considered a linear axisymmetric approach for the pure Marangoni and pure Rayleigh problems. They determined the critical Marangoni and Rayleigh numbers for the onset of convection in cylindrical containers with varying aspect ratios and compared their results with those of Charlson and Sani [20] obtained for buoyant convection in rigid-wall cylinders. Convective thresholds were also obtained by Wagner et al. [21] from nonlinear solutions extrapolated to zero amplitude. These thresholds appear to be in good agreement with those of Vrentas et al. [19]. Zaman and 
Narayanan [22] considered the three-dimensional linear Bénard-Marangoni problem for cylindrical cavities. They determined the critical Marangoni number at fixed Rayleigh number or at fixed ratio of these two parameters for the first four azimuthal eigenmodes. Some discrepancies are found with the experimental results of Koschmieder and Prahl [6], which are explained by sidewall induced imperfections and uncertainties in the viscosity of the test fluid. Dauby et al. [23] performed a numerical linear stability study in order to analyze the coupled influence of buoyancy and surface tension on the critical threshold in a real experiment. They show that the increase of the aspect ratio (radius/height) induces a change of the threshold, which is both due to less confinement and to a modification of the instability from buoyancy induced to surface tension induced. Recently, Assemat et al. [24] studied the effect of changing the container shape, from circular to elliptical, on the pattern formation in Marangoni convection for small aspect ratio containers.

In this paper, we consider a cylindrical cavity heated from below and with a free surface at the top. The problem corresponding to the Navier-Stokes equations coupled with an energy equation is solved by a spectral finite element method. An appropriate continuation method is used to study the nonlinear evolution of the convection beyond its onset, but also to follow the different bifurcation points and, in particular, the primary thresholds. Our purpose is to study the effect of the free surface (surface tension and heat exchange) on the buoyant convection. We will calculate stability diagrams giving the critical Rayleigh number $\mathrm{Ra}_{c}$, corresponding to the onset of the convection, as a function of the aspect ratio of the cavity $A$, the Marangoni number Ma, and the Biot number Bi. The nonlinear evolution of the convection beyond its onset will then be given through bifurcation diagrams for selected values of the parameters.

\section{PHYSICAL MODEL}

We consider an incompressible liquid layer contained in a vertical cylindrical cavity of aspect ratio $A=R_{d} / H$, where $R_{d}$ is the radius of the cavity and $H$ is its height (Fig. 1).

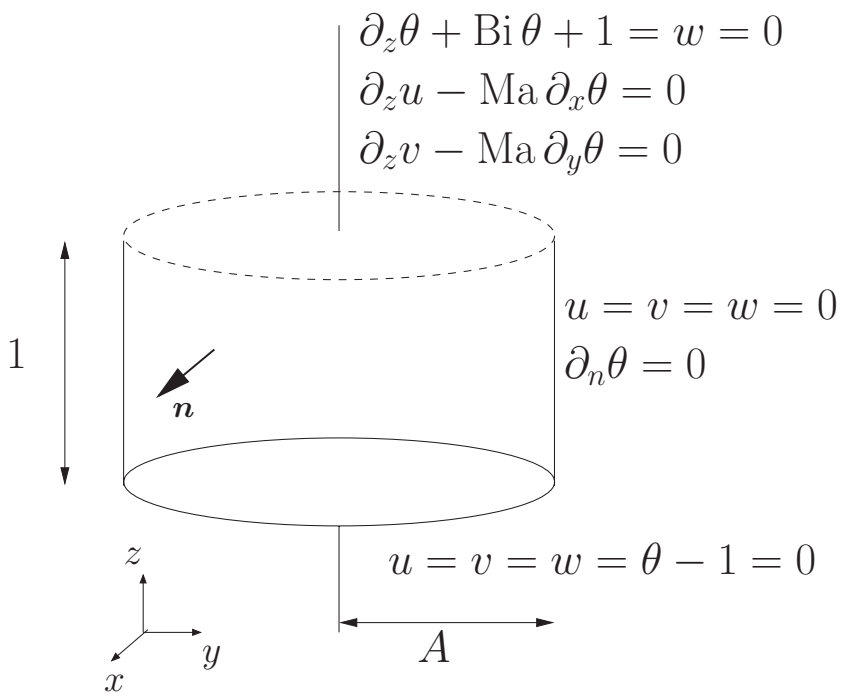

FIG. 1. Schematic diagram of the cylindrical cavity with the boundary conditions.
The lower end of the cylinder is assumed isothermal and held at the temperature $T_{b}$; a Newton law $-\lambda \partial_{z} T=h\left(T-T_{g}\right)$ expresses the heat exchange between the liquid free surface and the ambient gas ( $\lambda$ is the liquid thermal conductivity, $h$ the heat exchange coefficient, and $T_{g}$ the temperature of the ambient gas); the vertical sidewalls are considered as adiabatic. The fluid is assumed to be Newtonian with constant physical properties (kinematic viscosity $\nu$, thermal diffusivity $\kappa$, density $\rho$ ), except for the density in the buoyancy term, which, in the Boussinesq approximation, depends linearly on temperature $\rho=\rho_{\text {ref }}\left[1-\beta\left(T-T_{\text {ref }}\right)\right]$, where $\beta$ is the thermal expansion coefficient, $T_{\text {ref }}$ is a reference temperature, and $\rho_{\text {ref }}$ is the value of the density at $T_{\text {ref. }}$. The free surface is flat and subject to a surface tension $\sigma$, which is assumed to vary linearly with the temperature $\sigma=\sigma_{\text {ref }}\left[1+\gamma\left(T-T_{\text {ref }}\right)\right]$, where $\sigma_{\text {ref }}$ is the surface tension at $T_{\text {ref }}$ and $\gamma$ is constant.

The imposed thermal conditions generate a conductive rest state, which corresponds to a linear temperature profile along the vertical coordinate

$$
T(\bar{z})=-\frac{\mathrm{Bi}}{1+\mathrm{Bi}}\left(T_{b}-T_{g}\right) \frac{\bar{z}}{H}+T_{b},
$$

with a temperature at the upper surface of the layer $T_{t}=$ $-\mathrm{Bi}\left(T_{b}-T_{g}\right) /(1+\mathrm{Bi})+T_{b}$ and, thus, a temperature difference across the layer $\Delta T=T_{b}-T_{t}=\mathrm{Bi}\left(T_{b}-T_{g}\right) /(1+\mathrm{Bi})$. In these expressions, $\mathrm{Bi}$ is the Biot number $\mathrm{Bi}=h H / \lambda$.

Distance, time, and velocity are nondimensionalized by $H$, $H^{2} / \kappa$, and $\kappa / H$, respectively. Concerning temperature, we will use the scaling based on the temperature difference across the layer at rest $\Delta T$, and define the dimensionless temperature as $\theta=\left(T-T_{t}\right) / \Delta T$ and $T_{\text {ref }}$ as $T_{t}$ (this scaling is used, for example, by Dauby et al. [23]). The convective motion is governed by the Navier-Stokes equations coupled to an energy equation. In dimensionless form, these equations are

$$
\begin{gathered}
\nabla \cdot \boldsymbol{u}=0, \\
\partial_{t} \boldsymbol{u}+(\boldsymbol{u} \cdot \nabla) \boldsymbol{u}=-\nabla p+\operatorname{Pr} \nabla^{2} \boldsymbol{u}+\operatorname{Pr} \operatorname{Ra} \theta \boldsymbol{e}_{z}, \\
\partial_{t} \theta+(\boldsymbol{u} \cdot \nabla) \theta=\nabla^{2} \theta,
\end{gathered}
$$

where $e_{z}$ is the unit vector in the vertical direction. We impose no-slip boundary conditions along the rigid bottom and lateral walls. The lateral walls are insulating, while a constant temperature is maintained on the bottom. Along the rigid bottom at $z=0$, we thus obtain $u=v=w=0, \theta=1$, and along the lateral walls at $r=A, u=v=w=\partial_{r} \theta=0$. Along the free surface, the normal velocity $w$ is zero and the stress equilibrium gives the boundary conditions for $u$ and $v$, whereas the heat exchange law gives the boundary condition for $\theta$. At the free surface at $z=1$, we thus obtain

$$
\frac{\partial u}{\partial z}-\operatorname{Ma} \frac{\partial \theta}{\partial x}=\frac{\partial v}{\partial z}-\operatorname{Ma} \frac{\partial \theta}{\partial y}=\frac{\partial \theta}{\partial z}+\operatorname{Bi} \theta+1=w=0 .
$$

The nondimensional parameters arising from the scaling of the equations are the Rayleigh number $\mathrm{Ra}=\left(\beta g \Delta T H^{3} / \nu \kappa\right)$, 
the Prandtl number $\operatorname{Pr}=v / \kappa$, the Marangoni number $\mathrm{Ma}=$ $\left(\Delta T H \sigma_{\text {ref }} \gamma / \rho \nu \kappa\right)$, and the Biot number already defined. The dimensionless variables are the velocity vector $\boldsymbol{u}=(u, v, w)$ [defined in the $(x, y, z)$ coordinates], the pressure $p$, and the temperature $\theta$, and the dimensionless conductive temperature profile is given by $\theta(z)=1-z$.

We also want to mention another scaling for the temperature (used, for example, by Vrentas et al. [19]), which is based on the temperature difference between the bottom of the layer and the gas. In that case, the dimensionless temperature becomes $\theta^{\prime}=\left(T-T_{g}\right) /\left(T_{b}-T_{g}\right)=(1+\mathrm{Bi} \theta) /(1+\mathrm{Bi})$. This would give a conductive temperature profile $\theta^{\prime}(z)=1-\mathrm{Bi} z /(1+$ Bi) and a thermal boundary condition at the free surface $\partial_{z} \theta^{\prime}+$ $\operatorname{Bi} \theta^{\prime}=0$. The Rayleigh and Marangoni numbers would also be changed and could be written as $\mathrm{Ra}^{\prime}=\mathrm{Ra}(1+\mathrm{Bi}) / \mathrm{Bi}$ and $\mathrm{Ma}^{\prime}=\mathrm{Ma}(1+\mathrm{Bi}) / \mathrm{Bi}$. This scaling is equivalent to the first scaling for infinite $\mathrm{Bi}$ where the temperature at the upper surface of the layer is equal to the temperature of the ambient gas $\left(T_{t}=T_{g}\right)$. It differs from the first scaling for finite values of $\mathrm{Bi}$, particularly for $\mathrm{Bi}=0$, where it becomes inappropriate as it gives infinite values of the parameters $\mathrm{Ra}^{\prime}$ and $\mathrm{Ma}^{\prime}$ associated with a constant temperature in the layer, whereas the first scaling gives finite values of $\mathrm{Ra}$ and $\mathrm{Ma}$.

\section{NUMERICAL METHODS}

The governing equations are solved in the threedimensional cylindrical domain using a spectral element method as described in Ref. [25]. The domain is divided into five macroelements, and the fields in each element are discretized as a Lagrangian interpolant on Legendre-GaussLobatto points distributions. The time discretization is carried out using a semi-implicit splitting scheme where, as proposed by Karniadakis et al. [26], the nonlinear terms are first integrated explicitly, the pressure is then solved through a pressure equation enforcing the incompressibility constraint, and the linear terms are finally integrated implicitly. This time integration scheme is used for transient computations with the third-order accurate formulation described in Ref. [26]. In its first-order formulation, it is used for steady state solving (Mamun and Tuckerman [27]), eigenvalue and eigenvector calculation, and determination of bifurcation points (Henry and Ben Hadid [28]) through a Newton method.

The first-order time scheme can be written in the abbreviated form

$$
\frac{\boldsymbol{X}^{(n+1)}-\boldsymbol{X}^{(n)}}{\Delta t}=\mathcal{N}\left(\boldsymbol{X}^{(n)}, \mathrm{Ra}\right)+\mathcal{L} \boldsymbol{X}^{(n+1)},
$$

where $\boldsymbol{X}$ denotes all of the spatially discretized fields $(u, v, w, \theta)$ and $\mathcal{N}$ and $\mathcal{L}$ are the spatially discretized nonlinear and linear operators. For the purpose of Newton solver, this time scheme can be slightly modified and expressed as

$$
\boldsymbol{X}^{(n+1)}-\boldsymbol{X}^{(n)}=-\mathcal{L}^{-1}\left[\mathcal{N}\left(\boldsymbol{X}^{(n)}, \mathrm{Ra}\right)+\mathcal{L} \boldsymbol{X}^{(n)}\right]
$$

Now, we consider the steady state problem

$$
\mathcal{N}(\boldsymbol{X}, \mathrm{Ra})+\mathcal{L} \boldsymbol{X}=0
$$

and solve it with a Newton method. Each Newton step can be written as

$$
\begin{gathered}
{\left[\mathcal{N}_{\boldsymbol{X}}(\boldsymbol{X}, \mathrm{Ra})+\mathcal{L}\right] \delta \boldsymbol{X}=-[\mathcal{N}(\boldsymbol{X}, \mathrm{Ra})+\mathcal{L} \boldsymbol{X}]} \\
\boldsymbol{X} \leftarrow \boldsymbol{X}+\delta \boldsymbol{X},
\end{gathered}
$$

where $\mathcal{N}_{\boldsymbol{X}}(\boldsymbol{X}, \mathrm{Ra})$ is the Jacobian of $\mathcal{N}$ with respect to $\boldsymbol{X}$ evaluated at $\boldsymbol{X}$ and $\mathrm{Ra}$. In order to improve the convergence of the iterative inversion, we rather solve

$-\mathcal{L}^{-1}\left[\mathcal{N}_{\boldsymbol{X}}(\boldsymbol{X}, \mathrm{Ra})+\mathcal{L}\right] \delta \boldsymbol{X}=-\left(-\mathcal{L}^{-1}\right)[\mathcal{N}(\boldsymbol{X}, \mathrm{Ra})+\mathcal{L} \boldsymbol{X}]$,

where the operator $-\mathcal{L}^{-1}$ serves as a preconditioner (i.e., approximate inverse for $\mathcal{N}+\mathcal{L}$ ). If we solve the linear system (8) by an iterative conjugate gradient method, we need only provide the right-hand side and the action of the matrix-vector product constituting the left-hand side. Referring to Eq. (5), we see that the right-hand side of Eq. (8) can be obtained by carrying out a time step and the matrix-vector product by carrying out a linearized version of the same time step. The Jacobian matrix is, thus, never constructed or stored. The GMRES algorithm from the NSPCG software library [29] is used as iterative solver. This algorithm is memory consuming, but it was found to be more robust than the biconjugate gradient squared (BCGS) algorithms used previously by Touihri et al. [25], especially for the calculation of the bifurcation points.

When following a steady solution branch, we need to check the stability of the solutions. For that, we need to calculate leading eigenvalues, i.e., those with the largest real part and, thus, responsible for initiating instability, and their corresponding eigenvectors. To do so, we use Arnoldi's method from the ARPACK library and follow the ideas of Mamun and Tuckerman [27].

Finally, once we have identified a bifurcation point (the real part of an eigenvalue changed sign), we will be interested in locating it precisely (determination of the critical value of the continuation parameter) and then tracing its evolution as we vary other parameters of the problem. At such a bifurcation point, we have to write that $\boldsymbol{X}$ is a solution to Eq. (6) and that the Jacobian is singular, with a null vector $\boldsymbol{h}$. Here again, these equations are solved by a Newton method. At each Newton step, the preconditioned linear systems are solved iteratively using the GMRES conjugate gradient method, the different terms being evaluated through specifically adapted time steps.

Important information concerning the physical mechanisms involved in the flow transitions can be obtained from the calculation of the perturbation kinetic energy budget at critical parameter values. The basic steady solution $[u, v, w, \theta](x, y$, $z)$ or $\left.\left[u_{i}, \theta\right]\left(x_{i}\right)\right]$ and the critical eigenvector $\left[u^{\prime}, v^{\prime}, w^{\prime}, \theta^{\prime}\right](x$, $y, z)$ [or $\left.\left[u_{i}^{\prime}, \theta^{\prime}\right]\left(x_{i}\right)\right]$ both enter the equation of kinetic energy budget given by

$$
\frac{\partial K}{\partial t}=2 \lambda_{r} K=E_{\text {shear }}+E_{\text {visc }}+E_{\text {buoy }}+E_{\text {Mar }}
$$

where $\frac{\partial K}{\partial t}$ is the rate of change of the fluctuating kinetic energy defined as $K=\int_{\Omega}\left(u_{i}^{\prime} u_{i}^{\prime *} / 2\right) d \Omega, \lambda_{r}$ is the real part of the 
leading eigenvalue, and

$$
\begin{gathered}
E_{\text {shear }}=\operatorname{Re}\left(\int_{\Omega}-u_{j}^{\prime} \frac{\partial u_{i}}{\partial x_{j}} u_{i}^{\prime *} d \Omega\right), \\
E_{\text {visc }}=\operatorname{Re}\left(\int_{\Omega}-\operatorname{Pr} \frac{\partial u_{i}^{\prime}}{\partial x_{j}} \frac{\partial u_{i}^{\prime *}}{\partial x_{j}} d \Omega\right), \\
E_{\text {buoy }}=\operatorname{Re}\left(\int_{\Omega} \operatorname{Pr} \operatorname{Ra} \theta^{\prime} w^{\prime *} d \Omega\right), \\
E_{\text {Mar }}=\operatorname{Re}\left[\int_{S} \operatorname{Ma}\left(\frac{\partial \theta^{\prime}}{\partial x} u^{\prime *}+\frac{\partial \theta^{\prime}}{\partial y} v^{\prime *}\right) d S\right] .
\end{gathered}
$$

$E_{\text {shear }}$ represents the production of fluctuating kinetic energy by shear of the basic flow, $E_{\text {visc }}$ the viscous dissipation of fluctuating kinetic energy, $E_{\text {buoy }}$ the production of fluctuating kinetic energy by buoyancy, and $E_{\mathrm{Mar}}$ the production of fluctuating kinetic energy by the surface-tension forces. Re and the superscript $*$ denote the real part and the complex conjugate, respectively. $\Omega$ is the volume of the cylinder, whereas $S$ is the surface of the upper boundary. Note that $E_{\text {visc }}$ is stabilizing by nature and thus a negative term.

At threshold, the critical eigenvector is associated with an eigenvalue of zero real part. This implies that $\partial K / \partial t$ is equal to zero at marginal stability. The calculation of all the individual energy contributions enables us to determine which term plays a dominant role in triggering the instability through production of fluctuating kinetic energy. [Positive (negative) energy terms are destabilizing (stabilizing) respectively.] Finally, if we normalize Eq. (9) by $-E_{\text {visc }}=\left|E_{\text {visc }}\right|$, which is always positive, we can get another equation involving normalized energy terms $E^{\prime}=E /\left|E_{\mathrm{visc}}\right|$ at threshold:

$$
E_{\text {shear }}^{\prime}+E_{\text {buoy }}^{\prime}+E_{\text {Mar }}^{\prime}=1
$$

If we now consider primary thresholds, the basic flow is $\boldsymbol{u}=0$, which gives $E_{\text {shear }}^{\prime}=0$, and Eq. (10) is reduced to

$$
E_{\text {buoy }}^{\prime}+E_{\text {Mar }}^{\prime}=1 \text {. }
$$

Ra can also be factored out of the buoyancy energy term so that $E_{\text {buoy }}^{\prime}=\operatorname{Ra} E_{\text {buoy }}^{\prime \prime}$. Equation (11) at marginal stability can then be rewritten as

$$
\operatorname{Ra}_{c} E_{\text {buoy }}^{\prime \prime}=1-E_{\text {Mar }}^{\prime},
$$

which leads to

$$
\frac{\mathrm{Ra}_{c}}{\mathrm{Ra}_{c, 0}}=\overbrace{\left(\frac{E_{\text {buoy }, 0}^{\prime \prime}}{E_{\text {buoy }}^{\prime \prime}}\right)}^{R_{\text {buoy }}} \overbrace{\left(1-E_{\text {Mar }}^{\prime}\right)}^{R_{\text {mar }}},
$$

where the values with the subscript 0 refer to the case where $\mathrm{Ma}=0$. This decomposition of $\mathrm{Ra}_{c}$ will allow us to see whether variations of $\mathrm{Ra}_{c}$ with $\mathrm{Ma}$ are due to variations of $R_{\text {Mar }}$ directly connected to surface-tension effects or to variations of $R_{\text {buoy }}$ connected to modifications of the buoyancy contribution.

\section{RESULTS}

Tests of numerical accuracy have been performed. As it is shown in Table $\mathrm{I}$, a mesh corresponding to $\left(N_{x y} \times N_{z}\right)=$ $(193 \times 9)$ points enables a precise computation of the primary thresholds for the whole range of parameters considered. Concerning the nonlinear calculations, tests were performed on the values of the secondary bifurcation thresholds $\mathrm{Ra}_{S_{1}}$ and $\mathrm{Ra}_{S_{2}}$ (Table II). We see that a mesh corresponding to $(337 \times 11)$ points is now needed to have a precision for these thresholds about $1 \%$. Such a mesh has then been chosen for all the calculations. If we compare our results with those given by previous studies, we find a very good agreement with the more recently obtained results, as those of Dauby et al. [23] (Table III).

All the results presented in this paper have been obtained for a Prandtl number $\operatorname{Pr}=1$. We have first focused our study on the evolution of the critical Rayleigh number $\mathrm{Ra}_{c}$ for the onset of convection (primary thresholds) as a function of the different parameters of the problem, namely, the aspect ratio $A$, the Marangoni number $\mathrm{Ma}$, and the Biot number Bi. (Note that these primary thresholds do not depend on Pr when they are expressed as critical Rayleigh numbers.) We have then followed the evolution of the convective structures beyond the primary thresholds as a function of the Rayleigh number, and the results are presented as bifurcation diagrams.

\section{A. Onset of convection}

\begin{tabular}{|c|c|c|c|c|c|c|}
\hline & & & & \multicolumn{3}{|c|}{$\operatorname{Mesh}\left(N_{x y} \times N_{z}\right)$} \\
\hline \multicolumn{4}{|c|}{ Case } & $(89 \times 7)$ & $(193 \times 9)$ & $(337 \times 11)$ \\
\hline$A=1.5$ & $m=0$ & $\mathrm{Ma}=0$ & $\mathrm{Bi}=100$ & 1214.0 & 1212.6 & 1212.6 \\
\hline$A=1.5$, & $m=0$ & $\mathrm{Ma}=0$ & $\mathrm{Bi}=1$ & 886.7 & 885.3 & 885.3 \\
\hline$A=1.5$ & $m=1$ & $\mathrm{Ma}=100$ & $\mathrm{Bi}=1$ & 1591.5 & 1585.7 & 1585.7 \\
\hline$A=0.5$ & $m=1$ & $\mathrm{Ma}=100$ & $\mathrm{Bi}=1$ & 3104.7 & 3105.7 & 3106.0 \\
\hline
\end{tabular}

As it is well known, in our configuration, convection will set in above a critical threshold, and the way it will develop will depend on the symmetries of the system. The symmetries of the equations and boundary conditions in our cylindrical cavity with a free surface correspond to the group $O(2)$, which is

TABLE I. Tests of numerical accuracy: the critical Rayleigh numbers $\mathrm{Ra}_{c}$ for different primary bifurcations are given as a function of the mesh size. 
TABLE II. Tests of numerical accuracy: The secondary thresholds $\mathrm{Ra}_{S_{1}}$ and $\mathrm{Ra}_{S_{2}}$ of Fig. 12 are given as a function of the mesh size.

\begin{tabular}{lccc}
\hline \hline & \multicolumn{3}{c}{ Mesh $\left(N_{x y} \times N_{z}\right)$} \\
\cline { 2 - 4 } & $(193 \times 9)$ & $(337 \times 11)$ & $(521 \times 13)$ \\
\hline $\operatorname{Ra}_{S_{1}}$ & 9166 & 10112 & 10006 \\
$\mathrm{Ra}_{S_{2}}$ & 13493 & 12513 & 12615 \\
\hline \hline
\end{tabular}

generated by the rotations around the $z$ axis and the reflections with respect to the vertical planes containing this axis. These symmetries are also those of the basic conduction state. This $O(2)$ symmetry of the system and of the conduction state implies that the eigenmodes involved at the onset of convection will correspond to Fourier modes in the azimuthal direction [variation in $\exp (\operatorname{im} \phi)$, where $\phi$ is the azimuthal angle]. We will consider four of these modes (the most important), i.e., the axisymmetric mode $m=0$ and the first three asymmetric modes $m=1,2$, and 3 . At the primary bifurcations, the corresponding modes can be characterized by their broken symmetries with regard to the symmetries of the problem. In fact, the number of solutions obtained at a bifurcation can be deduced from the number of broken symmetries because every image of a solution by a broken symmetry is also a solution. For the axisymmetric mode, which keeps the $O(2)$ symmetry of the system, the bifurcation is transcritical, associated with a single critical eigenvalue, and it generates two different states belonging to a supercritical and to a subcritical branch. For the asymmetric modes $m=1,2$, and 3 , the rotational invariance is broken, and they keep reflection symmetries with respect to one, two, and three vertical planes containing the axis of the cylinder, respectively. For each of these modes, we thus have a symmetry-breaking bifurcation that generates an infinite number of equivalent solutions (defined to within a rotation). This type of bifurcation, which is associated with a double eigenvalue, is called a circular pitchfork bifurcation (or pitchfork of revolution). It is interesting to make a comparison with the case where the cylinder is a closed container (no-slip boundary condition applied at the top and bottom of the container). In that case, there is an extra symmetry, which is the up-down symmetry (reflection symmetry with respect to the horizontal midplane), and this symmetry is systematically broken at the onset of convection. This implies that the $m=0$ bifurcation becomes a pitchfork and that the $m=1$ mode has an extra symmetry with respect to the horizontal middle axis perpendicular to its symmetry plane.

\section{Influence of the aspect ratio}

We first consider the influence of the aspect ratio on the onset of convection. The stability diagrams given in Fig. 2 show the evolution of the first four primary thresholds corresponding to the modes $m=0,1,2$, and 3 , as a function of the aspect ratio $A$. Two cases have been chosen corresponding to $\mathrm{Bi}=100$ and $\mathrm{Ma}=0$ [Fig. 2(a)] and $\mathrm{Bi}=1$ and $\mathrm{Ma}=500$ [Fig. 2(b)]. As we shall see later, only the first (lowest $\operatorname{Ra}_{c}$ ) bifurcation leads directly to a stable flow. Subsequent (higher $\mathrm{Ra}_{c}$ ) bifurcations are responsible for the creation of branches that are initially unstable.

From these diagrams, we see that there is a global decrease of the thresholds when $A$ is increased (destabilization due to less lateral confinement), but this decrease is not monotonic. For each mode, there are zones of strong decrease followed by zones of smaller decrease or even small increase. Near the points where the decrease progresses again, the number of rolls changes via the creation of rolls that are initially infinitesimally small and that develop according to the symmetries of the mode. Minima or pseudominima indicate the range of aspect ratio where a given mode configuration can settle at best. The four Fourier modes have different symmetries, so the corresponding curves can cross. These crossings change the order of appearance of the modes and can also change the type of modes corresponding to the first bifurcation. In Fig. 2, we see that, for the long cylinders ( $A$ roughly smaller than 1 ), convection sets in as an $m=1$ mode, whereas for shorter cylinders, different modes will be successively involved, the first three corresponding to an $m=0$ mode, $m=1$ mode, and $m=2$ mode.

The values of $A$ at which the transitions between the modes occur are larger for $\mathrm{Bi}=100$ and $\mathrm{Ma}=0$ [Fig. 2(a)] than for $\mathrm{Bi}=1$ and $\mathrm{Ma}=500$ [Fig. 2(b)]. The first two transitions (from $m=1$ to 0 and from $m=0$ to 1 ) occur for $A=1.03$ and 1.85 in the first case and $A=0.875$ and 1.375 in the second case, and the transition to the $m=2$ mode is changed from $A \approx 2.4$ to $A \approx 1$.9. These modifications of the transition points occur because the preferred roll size is different in the two cases (smaller in the second case). Finally, for large values of $A$ [as $A \gtrsim 2.5$ in Fig. 2(b)], the four modes have very close thresholds and there will be successive transitions to the different modes at nearly the same value of $\mathrm{Ra}_{c}$. The thresholds $\mathrm{Ra}_{c}$ for the different modes eventually tend to a limiting value for infinite aspect ratio, but the approach of this limit is not monotonic but slightly wavy. For $\mathrm{Bi}=100$ and $\mathrm{Ma}=0$, this limit of $\mathrm{Ra}_{c}$ is close to 1100 , a value obtained in a layer with a rigid bottom, a free top surface (without surface tension), and prescribed temperatures at these two surfaces (equivalent to $\mathrm{Bi} \rightarrow \infty)[30]$.

TABLE III. Validation tests: the critical Rayleigh numbers $\mathrm{Ra}_{c}$ for different primary bifurcations are compared with results found in the literature. The mesh used for our calculations is $337 \times 11$.

\begin{tabular}{llllcr}
\hline \hline & \multicolumn{2}{c}{ Case } & Vrentas et al. [19] & Dauby et al. [23] & Current work \\
\hline$A=1$, & $m=0$, & $\mathrm{Ma}=0$, & $\mathrm{Bi}=0.1$ & 1565.9 & 1426.2 \\
$A=1$, & $m=0$, & $\mathrm{Ma}=0$, & $\mathrm{Bi}=1$ & 1628.2 & 1482.1 \\
$A=1$, & $m=0$, & $\mathrm{Ma}=0$, & $\mathrm{Bi}=100$ & 1938.6 & 1481.8 \\
\hline \hline
\end{tabular}




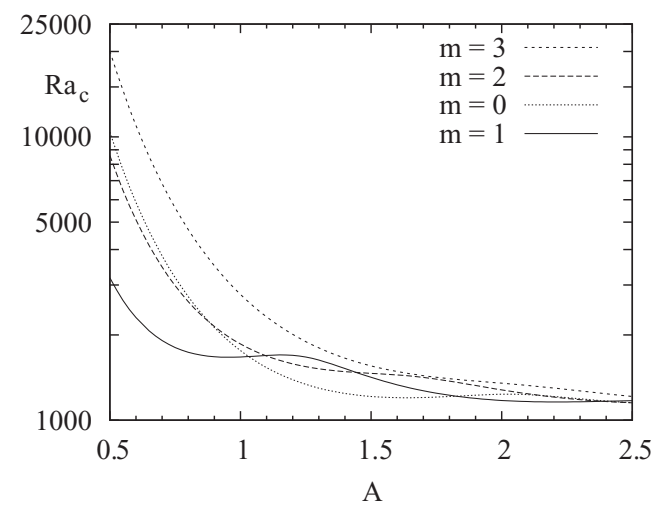

(a)

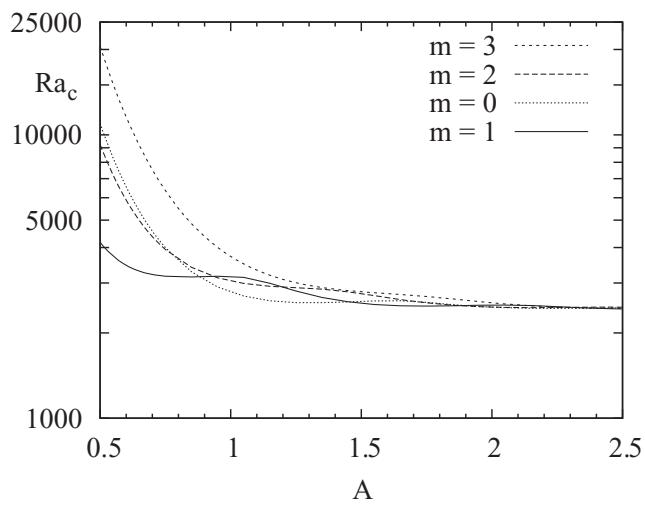

(b)

FIG. 2. Variation of the first primary thresholds $\mathrm{Ra}_{c}$ corresponding to the modes $m=0,1,2$, and 3 as a function of the aspect ratio $A$ : (a) $\mathrm{Bi}=100$ and $\mathrm{Ma}=0$; (b) $\mathrm{Bi}=1$ and $\mathrm{Ma}=500$.

The flow structures for the modes $m=1$ and 0 , which are dominant in large ranges of $A$, are plotted in Figs. 3 and 4, respectively. The mode $m=1$ is given for $A=0.75$ and the mode $m=0$ for $A=1.2$. In both cases, the two situations $(\mathrm{Bi}=100, \mathrm{Ma}=0)$ and $(\mathrm{Bi}=1, \mathrm{Ma}=500)$ are considered. As shown in Fig. 3(a) (vertical velocity at midheight in the cylinder), the flow for the mode $m=1$ is up on one half of the cylindrical section and down on the other half. For $\mathrm{Bi}=$ 100 and $\mathrm{Ma}=0$ [Fig. 3(b)], this corresponds to a convective cell occupying the whole height of the cavity. In contrast, for $\mathrm{Bi}=1$ and $\mathrm{Ma}=500$ [Fig. 3(c)], the main convective cell is surmounted by a smaller counter-rotating cell and the flow at the upper surface has changed direction. (Note that the flow for the mode $m=1$ is defined to within an azimuthal rotation so that a particular orientation of the flow has to be chosen for the plots. We have chosen to give flow structures oriented along the $x$ and $y$ axes.) The flow for the mode $m=0$ is axisymmetric, with either upflow (Fig. 4) or downflow at the center along the cylinder axis and a reverse flow along the lateral boundaries. For $\mathrm{Bi}=100$ and $\mathrm{Ma}=0$ [Fig. 4(b)], this corresponds to a toroidal convective cell occupying the whole height of the cavity. In contrast, for $\mathrm{Bi}=1$ and $\mathrm{Ma}=500$ [Fig. 4(c)], the main toroidal roll is surmounted by a smaller counter-rotating toroidal roll. For both $m=1$ and 0 modes, it is thus observed that the vertical extent of the main convective rolls is reduced in the case $\mathrm{Bi}=1$ and $\mathrm{Ma}=500$. This can explain that the preferred roll size is smaller in this case than in the case $\mathrm{Bi}=100$ and $\mathrm{Ma}=0$.

\section{Influence of the Biot and Marangoni numbers}

We now turn to the influence of the Biot and Marangoni numbers on the onset of convection. Figure 5(a) depicts the dependence of the critical Rayleigh number $\mathrm{Ra}_{c}$ on the Biot number Bi. We plot Rayleigh numbers corresponding to the critical axisymmetric $m=0$ mode in the cavity with aspect ratio $A=1.5$ for different values of $\mathrm{Ma}$.

We see that the influence of $\mathrm{Bi}$ on the convection thresholds is monotonic, inducing either an increase or a decrease of the critical Rayleigh number when $\mathrm{Bi}$ is increased. An increase is obtained for $\mathrm{Ma} \leqslant 50$, and this increase becomes steeper as $\mathrm{Ma}$ is decreased; a decrease is obtained for $\mathrm{Ma} \geqslant 100$, and this

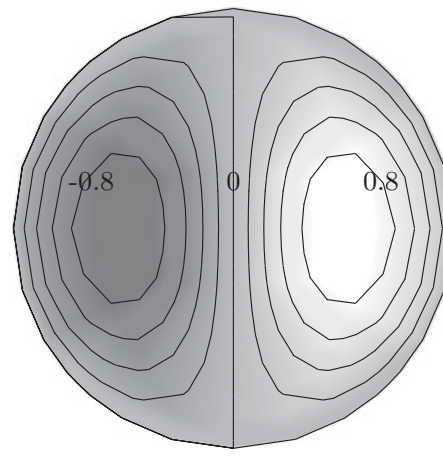

(a)

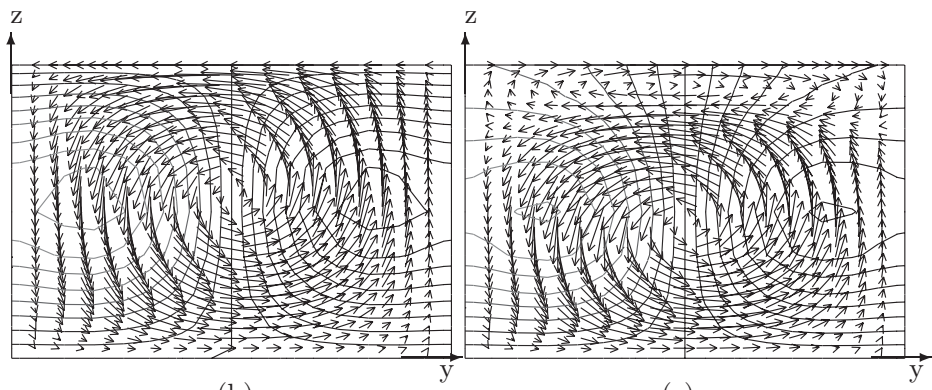

(c)

FIG. 3. Flow structure for the critical mode $m=1$ at threshold for $A=0.75$. (a) Vertical velocity contours at midheight and (b) velocity field and temperature contours in the vertical plane along $y$ for $\mathrm{Bi}=100$ and $\mathrm{Ma}=0$. (c) Same picture as in $(\mathrm{b})$ for $\mathrm{Bi}=1$ and $\mathrm{Ma}=500$. The modes are normalized by $w_{\max }$. For the velocity contours, dark (light) shading indicates $w<0(w>0)$. The step between the velocity contours is 0.2 . The step between the temperature isolines is $5 \times 10^{-3}: 0$ is on the lower boundary, and on the isolines intersecting this boundary, the isolines evolve from black (stronger values) to gray (smaller values). 


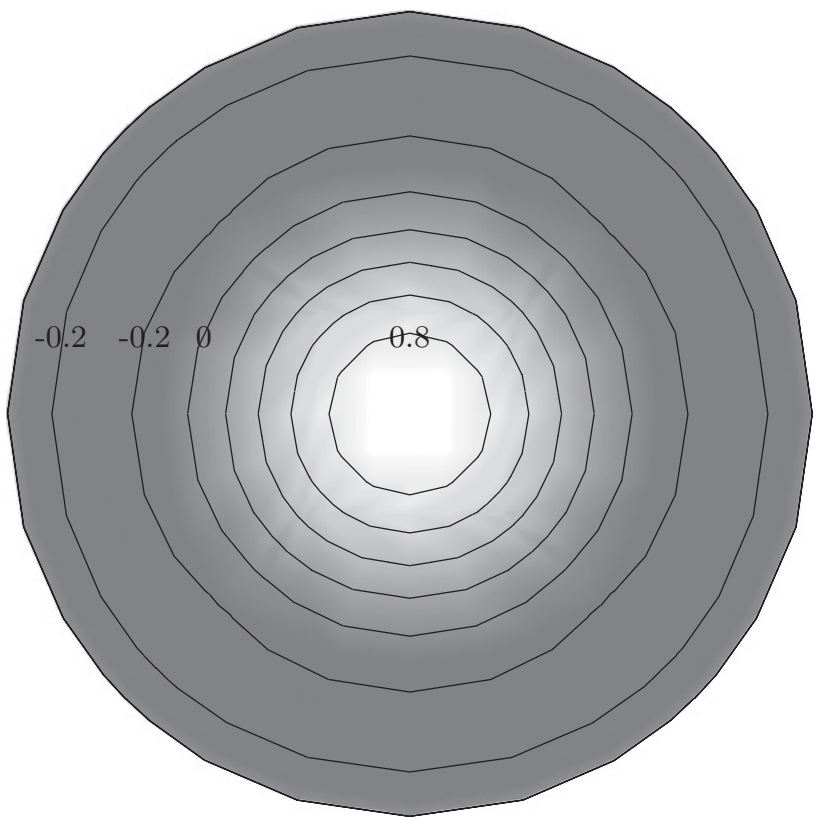

$\mathrm{Z}$

4

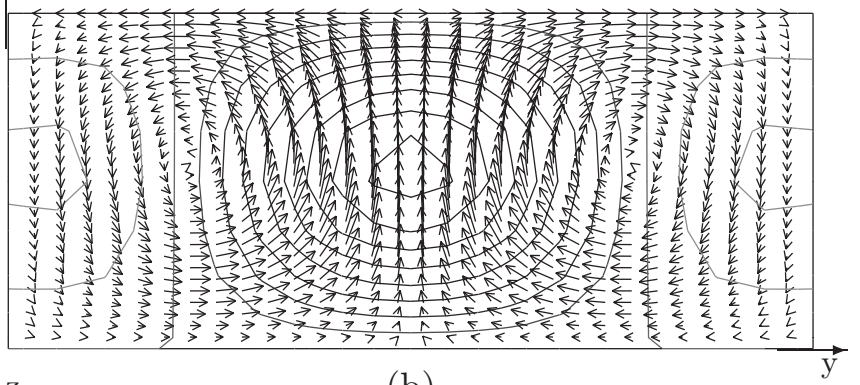

$\mathrm{Z}$

(b)

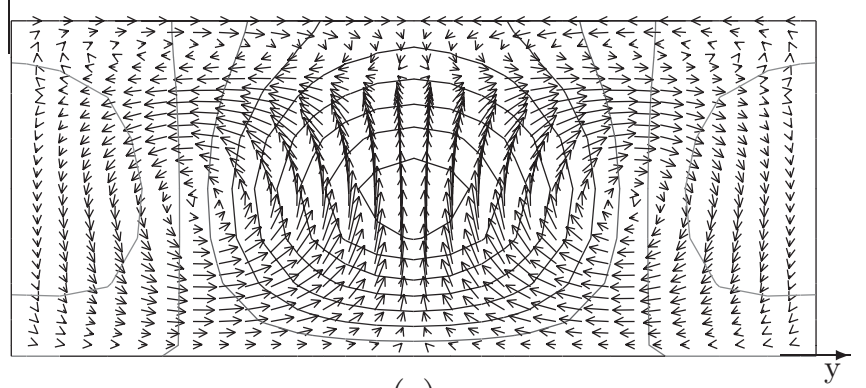

(c)

FIG. 4. Flow structure for the critical mode $m=0$ at threshold for $A=1.2$. (a) Vertical velocity contours at midheight and (b) velocity field and temperature contours in a centered vertical plane for $\mathrm{Bi}=$ 100 and $\mathrm{Ma}=0$. (c) Same picture as in (b) for $\mathrm{Bi}=1$ and $\mathrm{Ma}=$ 500 . The modes are normalized by $w_{\max }$. For the velocity contours, dark (light) shading indicates $w<0(w>0)$. The step between the velocity contours is 0.2 . The step between the temperature isolines is $5 \times 10^{-3}$ : 0 is on the lower boundary, and on the isolines intersecting this boundary, the isolines evolve from black (stronger values) to gray (smaller values).

decrease becomes steeper as Ma is increased. In the range $50 \leqslant$ $\mathrm{Ma} \leqslant 100$, we expect to find a value $\mathrm{Ma}_{B}$ of $\mathrm{Ma}$ for which the Biot number will have no influence. Further calculations have allowed us to approximate this value to $\mathrm{Ma}_{B}=65.38$. The corresponding threshold is $\mathrm{Ra}_{c} \approx 1230$ with a variation with Bi smaller than $0.2 \%$. All the critical curves eventually collapse for $\mathrm{Bi} \rightarrow \infty$ and approach a limiting value of $\mathrm{Ra}_{c}$ close above 1200. Similar variations of the thresholds with the Biot number are obtained for other modes, as for example the $m=0$ and 1 modes for the cavity with aspect ratio $A=1$ and the $m=1$ mode for the cavity with aspect ratio $A=0.75$.

To have a better understanding of the influence of the Marangoni number on the onset of convection, we now show the dependence of the critical Rayleigh number $\mathrm{Ra}_{c}$ on the Marangoni number Ma in Fig. 5(b). The critical curves are given for four values of the Biot number $(\mathrm{Bi}=0,1,10$, and $100)$ in the case $A=0.75$ and for the critical asymmetric $m=1$ mode. All four curves increase monotonically with $\mathrm{Ma}$, with this increase being the largest for $\mathrm{Bi}=0$ and quite small for $\mathrm{Bi}=100$. The four curves intersect in the vicinity of $\mathrm{Ma}=100$ for $\mathrm{Ra}_{c} \approx 1830$. This means that the convection thresholds do not depend on $\mathrm{Bi}$ for this particular value of $\mathrm{Ma}$, and confirms what was obtained for the $m=0$ mode in the case $A=1.5$ [Fig. 5(a)]. This value $\mathrm{Ma}_{B}$ delimits two zones, a zone for larger values of $\mathrm{Ma}$ where the increase of $\mathrm{Bi}$ is destabilizing and a zone for smaller values of Ma where the increase of $\mathrm{Bi}$ is stabilizing. If we now consider the influence of $\mathrm{Ma}$ in comparison with the case without surface-tension effect $(\mathrm{Ma}=0)$, we see that positive values of Ma are stabilizing, leading to stronger values of the convection thresholds $\mathrm{Ra}_{c}$, whereas negative values of $\mathrm{Ma}$ are destabilizing, leading to smaller values of $\mathrm{Ra}_{c}$. $\mathrm{Ra}_{c}$ eventually goes to zero at a negative critical value of $\mathrm{Ma}\left(\mathrm{Ma}_{c}\right)$ depending on the value of $\mathrm{Bi}$ and corresponding to a pure Marangoni situation. Finally, for negative values of $\mathrm{Ma}$ below $\mathrm{Ma}_{c}$, the onset of convection is obtained for negative values of $\mathrm{Ra}_{c}$, i.e., in situations with stable thermal stratification.

We have seen that the influence of the surface tension (through the Marangoni number Ma) is strong for the small values of $\mathrm{Bi}$, whereas it is weak for the large values of $\mathrm{Bi}$. In order to explain this, we have to remember that the surfacetension effect is driven by the temperature perturbations at the free surface, and that these perturbations depend on the Biot number $\mathrm{Bi}$ and are very weak for the large values of $\mathrm{Bi}$ where the temperature at the free surface is nearly frozen to 0 , whereas they can develop more freely for the small values of $\mathrm{Bi}$ where a flux condition is applied.

We now give some comments on the variation with $\mathrm{Ma}$ of the $\mathrm{Bi}$ influence. For $\mathrm{Ma}=0$, there is no surface-tension effect, but the thermal boundary conditions at the free surface will affect the temperature perturbations in the bulk: small $\mathrm{Bi}$ values will favor these perturbations and will have then a destabilizing effect. For negative values of Ma, surface tension has a destabilizing effect, which is favored by the small values of $\mathrm{Bi}$ : this reinforces the destabilizing influence of the small $\mathrm{Bi}$ values. Finally, for positive values of Ma, two opposite effects are involved: small Bi values will favor both the destabilization by buoyancy and the stabilization by surface tension. As a consequence, for increasing $\mathrm{Ma}$, the initial destabilizing influence of the small $\mathrm{Bi}$ values will decrease until $\mathrm{Ma}_{B}$, where the Biot number $\mathrm{Bi}$ has no more influence on the onset of convection. Beyond $\mathrm{Ma}_{B}$, small $\mathrm{Bi}$ values will have a stabilizing influence because the increase of the temperature 


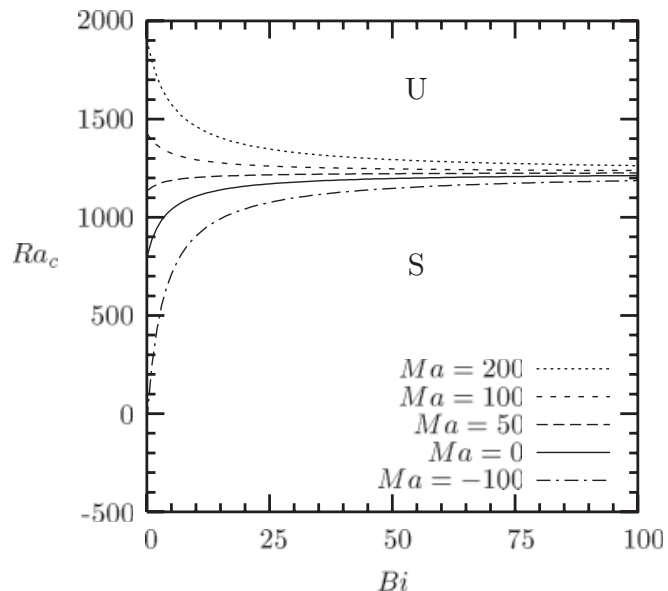

(a)

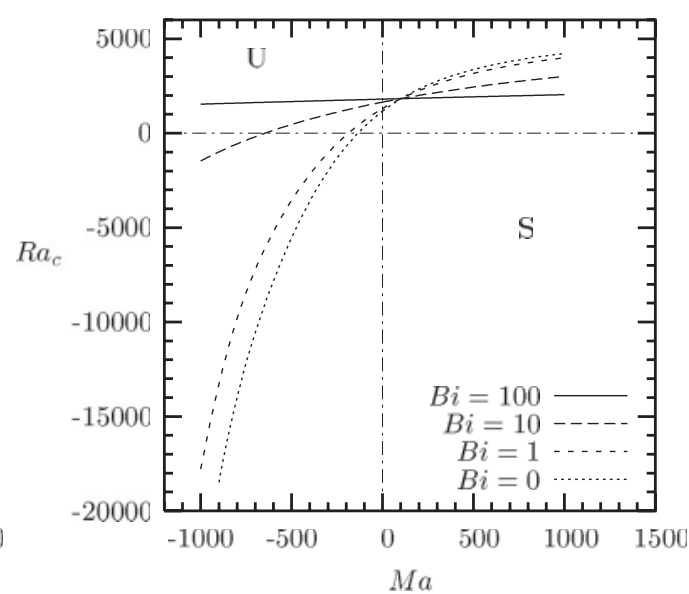

(b)

FIG. 5. Variation of the critical Rayleigh number $\mathrm{Ra}_{c}$ as a function of (a) the Biot number for different values of Ma and (b) the Marangoni number for various Bi. In the case (a), the aspect ratio is set to $A=1.5$ and the convection thresholds correspond to an $m=0$ mode, and in (b), $A=0.75$ and the thresholds correspond to an $m=1$ mode. $U$ denotes an unstable domain and $S$ a stable domain.

perturbations will predominantly promote the stabilization by surface tension.

\section{Energy analysis at the primary thresholds}

To gain insight into the action of surface tension on the convection thresholds, energy analyses at thresholds were performed for varying values of the Marangoni and Biot parameters. According to the results shown in the previous section, surface tension has a similar influence for cavities with different aspect ratios. Without loss of generality, we then focused our study on the case $A=1.5$ for which the critical mode is an axisymmetric $m=0$ mode.

The three energy terms contributing to the kinetic energy budget at the convection threshold [buoyancy term $E_{\text {buoy }}^{\prime}$, surface tension term $E_{\mathrm{Mar}}^{\prime}$, and viscous dissipation term normalized to -1, as shown in Eq. (11)] were calculated from the basic flow solution and critical eigenvector. The energy contributions $E_{\text {buoy }}^{\prime}$ and $E_{\mathrm{Mar}}^{\prime}$ are shown as a function of $\mathrm{Bi}$ for different values of Ma in Fig. 6(a) and as a function of Ma for different values of $\mathrm{Bi}$ in Fig. 6(b).

We first consider the influence of $\mathrm{Bi}$ on the energy contributions [Fig. 6(a)]. For Ma $=0$, the energy terms $E_{\text {buoy }}^{\prime}$ and $E_{\mathrm{Mar}}^{\prime}$ are 1 and 0 , respectively (dotted lines). Such values are also expected for any value of $\mathrm{Ma}$ for $\mathrm{Bi} \rightarrow \infty$ as, in this limit, the temperature at the free surface is frozen to 0 , without any horizontal gradient to generate surface tension forces. We see that, for large values of $\mathrm{Bi}$ (as $\mathrm{Bi}>60$, for example), $E_{\mathrm{Mar}}^{\prime}$ has a positive destabilizing contribution for $\mathrm{Ma}<0$ and a negative stabilizing contribution for $\mathrm{Ma}>0$. These contributions are those usually expected to explain the influence of surface tension on the stability of this convective situation. We can first remark that these contributions $E_{\text {Mar }}^{\prime}$ do not quickly tend to 0 for increasing $\mathrm{Bi}$ (as those for $E_{\text {buoy }}^{\prime}$ do not quickly tend to 1 ), and the values for $\mathrm{Bi}=100$ are still far from the asymptotic values. Moreover, for small values of $\mathrm{Bi}$, the energy contributions drastically change: the Marangoni

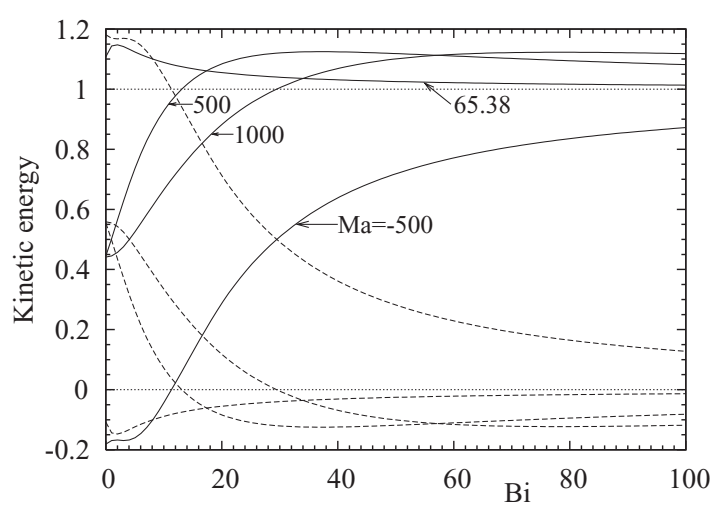

(a)

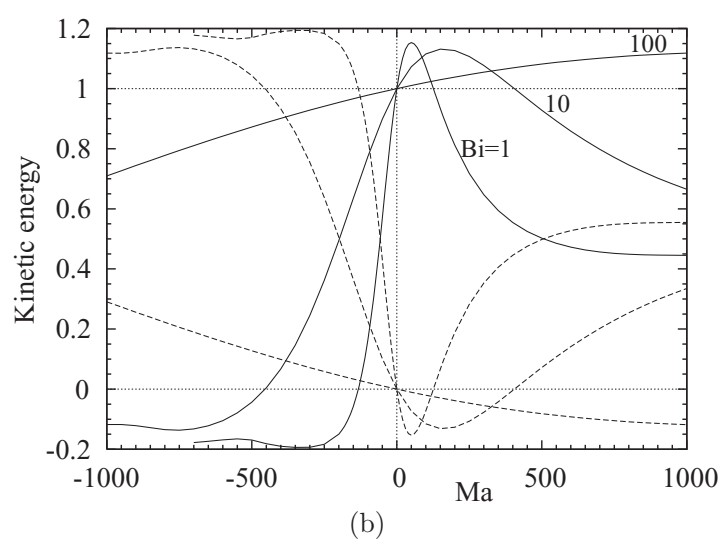

FIG. 6. Kinetic energy budget associated with the critical mode $m=0$ at threshold in a cylinder heated from below with a free surface ( $A=1.5$ ). The contributions normalized by the viscous dissipation energy are given in (a) as a function of the Biot number for different values of Ma and in (b) as a function of the Marangoni number for various $\mathrm{Bi}$. Solid lines represent the contribution of buoyancy $E_{\text {buoy }}^{\prime}$ and dashed lines the contribution of surface tension $E_{\mathrm{Mar}}^{\prime}$. Dotted lines represent the constant values 1 and 0 , which are also the buoyant and surface-tension contributions, respectively, for $\mathrm{Ma}=0$. 


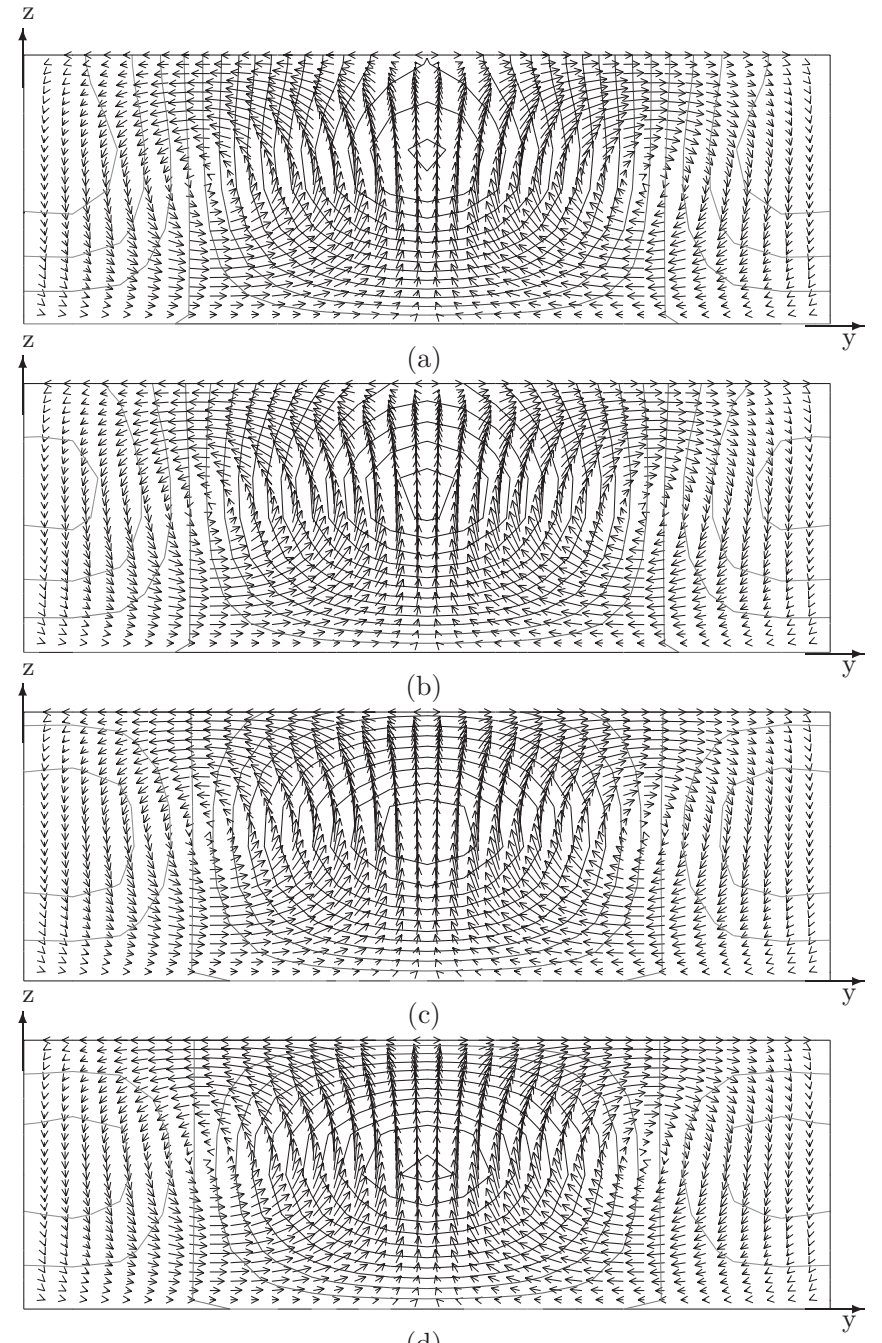

(d)

FIG. 7. Flow structure and temperature contours in a centered vertical plane for the critical mode $m=0$ at threshold for $A=1.5$ and $\mathrm{Ma}=65.38$ (this value of Ma gives a threshold that does not depend on $\mathrm{Bi}$ ). (a) $\mathrm{Bi}=0$; (b) $\mathrm{Bi}=1$; (c) $\mathrm{Bi}=10$; (d) $\mathrm{Bi}=100$. The modes are normalized by $w_{\max }$. The step between the temperature isolines is $5 \times 10^{-3}$ : 0 is on the lower boundary, and on the isolines intersecting this boundary, the isolines evolve from black (stronger values) to gray (smaller values).

contribution $E_{\mathrm{Mar}}^{\prime}$ becomes destabilizing for large enough positive values of $\mathrm{Ma}$, whereas the buoyancy contribution $E_{\text {buov }}^{\prime}$ can become negative for negative values of Ma. Note finally that, for $\mathrm{Ma}=\mathrm{Ma}_{B}=65.38$ (the case where $\mathrm{Ra}_{c}$ does not depend on $\mathrm{Bi}$ ), the Marangoni contribution $E_{\mathrm{Mar}}^{\prime}$ is always negative and globally increases in intensity as $\mathrm{Bi}$ is decreased. The flow structure for this value $\mathrm{Ma}_{B}$ is shown in Fig. 7 for different values of $\mathrm{Bi}(\mathrm{Bi}=0,1,10$, and 100$)$. We see that the convection rolls are really similar for the different values of $\mathrm{Bi}$, whereas the temperature perturbation fields are much changed due to the change of thermal boundary conditions at the upper surface. Compared to the case at $\mathrm{Bi}=100$ where the buoyant forces are principally responsible for the instability, smaller values of $\mathrm{Bi}$ induce destabilizing surface tension forces generated by horizontal temperature gradients at the upper surface, but also lead to a decrease of the buoyant forces in the cavity. According to Eq. (12), as the convection threshold is unchanged, the decrease of $1-E_{\mathrm{Mar}}^{\prime}$ due to the increase of $E_{\text {Mar }}^{\prime}$ is compensated by a similar decrease of $E_{\text {buoy }}^{\prime \prime}$.

To better understand what occurs at small $\mathrm{Bi}$, it is necessary to analyze the variations of the energy contributions with Ma, which are shown in Fig. 6(b). The variations for $\mathrm{Bi}=100$, already described, are given as a reference. For smaller values of $\mathrm{Bi}$ (as $\mathrm{Bi}=10$ and 1 ), we still get a negative stabilizing contribution of the Marangoni term $E_{\mathrm{Mar}}^{\prime}$ as $\mathrm{Ma}$ is increased from zero. This contribution, however, rapidly levels off, decreases in intensity, and becomes positive at a value of $\mathrm{Ma}$ denoted as $\mathrm{Ma}_{I}$, indicating a change to destabilization. This change occurs for smaller Ma values when $\mathrm{Bi}$ is decreased: $\mathrm{Ma}_{I}=1000$ for $\mathrm{Bi} \approx 29.45, \mathrm{Ma}_{I} \approx 405$ for $\mathrm{Bi}=10$, and $\mathrm{Ma}_{I} \approx 126$ for $\mathrm{Bi}=1 . E_{\mathrm{Mar}}^{\prime}$, now destabilizing, increases with $\mathrm{Ma}$ and seems to reach an asymptotic value at high $\mathrm{Ma}$ (as, for example, $E_{\mathrm{Mar}}^{\prime} \approx 0.55$ for $\mathrm{Bi}=1$ ). When $\mathrm{Ma}$ is decreased negatively, the Marangoni term $E_{\mathrm{Mar}}^{\prime}$ is, as expected, destabilizing. For small values of $\mathrm{Bi}$, this term strongly increases and reaches 1 ( $E_{\text {buoy }}^{\prime}$ is thus 0 ) in the pure Marangoni situation $\left(\operatorname{Ra}_{c}=0\right)$. Beyond this point, $E_{\mathrm{Mar}}^{\prime}$ still increases, but quickly levels off and tends to an asymptotic value.

The change of the energetic Marangoni contribution $E_{\mathrm{Mar}}^{\prime}$ from stabilizing to destabilizing at $\mathrm{Ma}_{I}$ in the positive $\mathrm{Ma}$ range is due to a flow inversion at the upper free surface. This is shown in Fig. 8, where the flow structures for the critical mode $m=0$ at threshold for $A=1.5$ and $\mathrm{Ma}=1000$ are plotted for $\mathrm{Bi}=1$ and 100 . For $\mathrm{Bi}=100$, the Marangoni contribution remains stabilizing for positive $\mathrm{Ma}$ and the flow structure plotted in Fig. 8(b) for Ma $=1000$ is the usual structure with

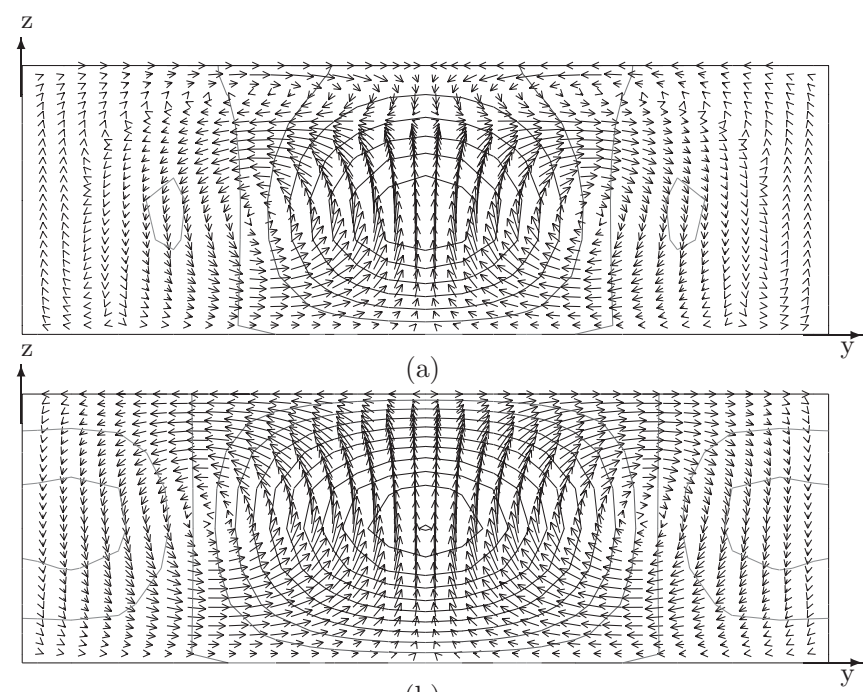

(b)

FIG. 8. Flow structure and temperature contours in a centered vertical plane for the critical mode $m=0$ at threshold for $A=1.5$ and $\mathrm{Ma}=1000$. (a) $\mathrm{Bi}=1$ : a counter-rotating toroidal cell appears in the upper part of the cavity and the main toroidal roll is compressed. (b) $\mathrm{Bi}=100$ : only the main roll is present. The modes are normalized by $w_{\max }$. The step between the temperature isolines is $5 \times 10^{-3}: 0$ is on the lower boundary, and on the isolines intersecting this boundary, the isolines evolve from black (stronger values) to gray (smaller values). 


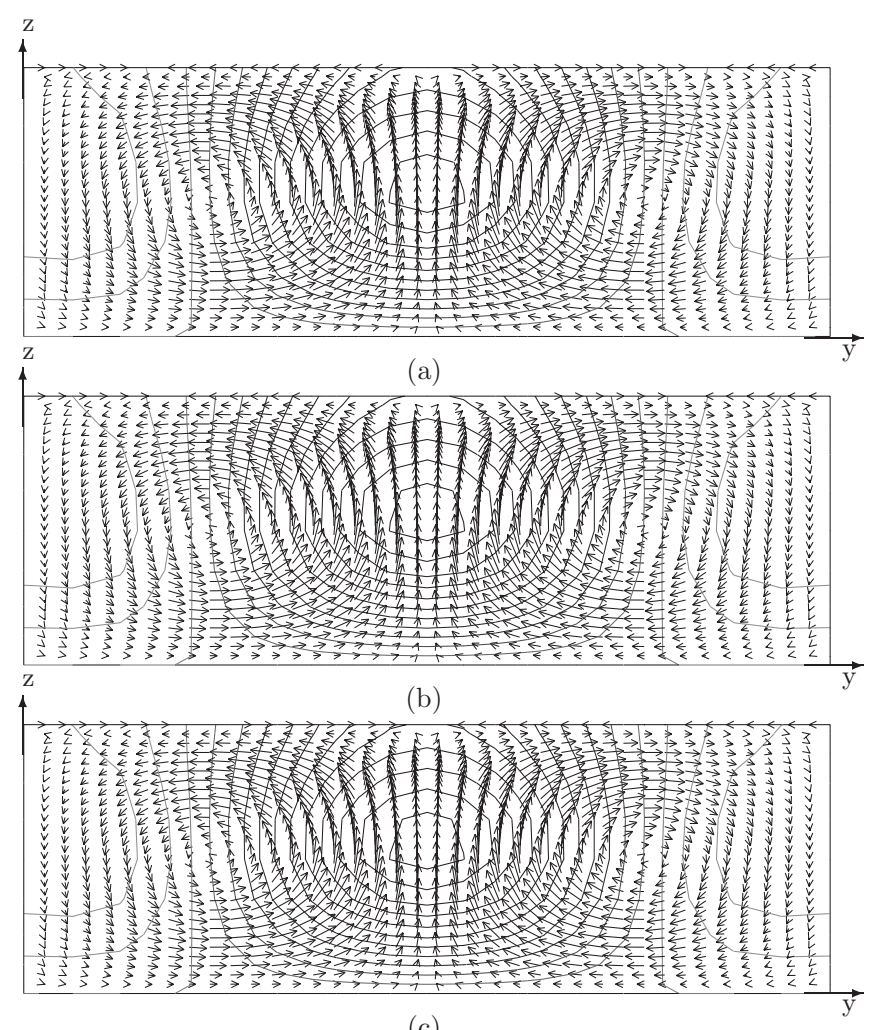

(c)

FIG. 9. Flow structure and temperature contours in a centered vertical plane for the critical mode $m=0$ at threshold for $A=1.5$, $\mathrm{Bi}=1$, and different values of Ma close to the value where there is a flow inversion at the upper boundary. (a) $\mathrm{Ma}=125$; (b) $\mathrm{Ma}=126$; (c) $\mathrm{Ma}=127$. The modes are normalized by $w_{\max }$. The step between the temperature isolines is $5 \times 10^{-3}$ : 0 is on the lower boundary, and on the isolines intersecting this boundary, the isolines evolve from black (stronger values) to gray (smaller values).

a large toroidal convective cell occupying the whole height of the cavity. In contrast, for $\mathrm{Bi}=1$, there is a change of $E_{\mathrm{Mar}}^{\prime}$ from stabilizing to destabilizing at $\mathrm{Ma}_{I} \approx 126$, and in the flow structure plotted in Fig. 8(a) for $\mathrm{Ma}=1000$, there appears a new counter-rotating toroidal cell in the upper part of the cavity. It is the flow inversion induced at the free surface, combined with a temperature field deformation that remains connected to the main flow, that explains the change of $E_{\mathrm{Mar}}^{\prime}$.

The phenomenon of flow inversion is highlighted in Figs. 9 and 10 for the critical mode $m=0$ at the threshold for $A=1.5$ and $\mathrm{Bi}=1$. Figure 9 gives the flow structure for Ma values $\left(\mathrm{Ma}=125,126\right.$, and 127) close to $\mathrm{Ma}_{I}$. We see that, in this Ma range, the temperature field remains almost unchanged. In contrast, the velocity at the free surface evolves with Ma. For $\mathrm{Ma}=125$, the velocity is mainly in the direction induced by buoyancy, but we already see a zone of flow inversion near the lateral wall. This zone is a little larger for $\mathrm{Ma}=126$, and for $\mathrm{Ma}=127$, it covers the whole free surface. More details on the flow inversion are given by the plot of the radial surface velocity along the radius in Fig. 10 . For $\mathrm{Ma}=0$, the radial surface velocity is positive, i.e., in the direction induced by buoyancy, all along the radius, and the maximum values reached (nearly $0.8 w_{\max }$ ) are quite strong. For $\mathrm{Ma}=50$, the radial velocity profile is similar, but the intensity is reduced by a factor of 2 . The beginning of flow inversion, which occurs along the lateral wall, is observed in our calculations for $\mathrm{Ma} \approx 80$. For increasing Ma up to $\mathrm{Ma}=125$, the intensity of the free-surface flow strongly decreases and the zone with inversed flow increases in size. At $\mathrm{Ma}=126$, a new zone of flow inversion appears close to the axis of the cylinder, and at $\mathrm{Ma}=127$, the flow inversion is effective on the whole free surface. Finally, for larger Ma, the inverse flow (negative radial velocities) will increase in intensity, initially with two velocity peaks and beyond $\mathrm{Ma}=140$ with a single peak.

Finally, in order to deepen our understanding of the stabilization effect found for positive $\mathrm{Ma}$, we use the $\mathrm{Ra}_{c}$ decomposition (based on the energy budget) given in Eq. (13). The two energetic contributions $R_{\text {buoy }}$ and $R_{\text {Mar }}$, which are such that $\mathrm{Ra}_{c} / \mathrm{Ra}_{c, 0}=R_{\text {buoy }} R_{\text {Mar }}$ and are both equal to 1 for $\mathrm{Ma}=0$, are given as a function of $\mathrm{Ma}$ for $\mathrm{Bi}=1$ and 100 in Fig. 11. We first see that $R_{\text {buoy }}$, which is 1 for $\mathrm{Ma}=0$, has values stronger than 1 when Ma is different from zero. This is observed both for small $(\mathrm{Bi}=1)$ and large $(\mathrm{Bi}=100)$ values of the Biot number, but the values reached by $R_{\text {buoy }}$ are much

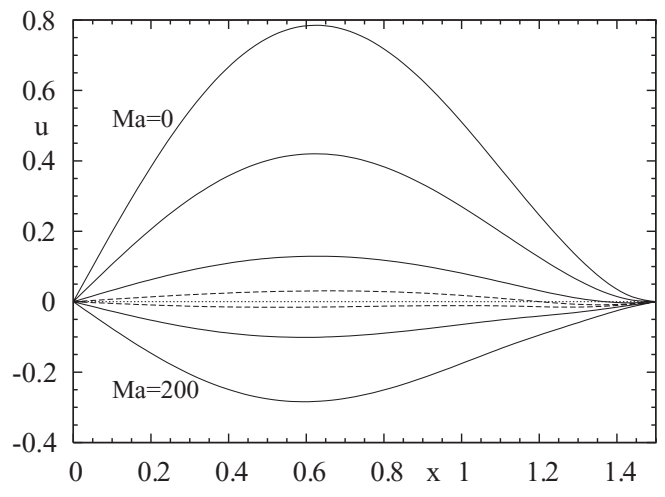

(a)

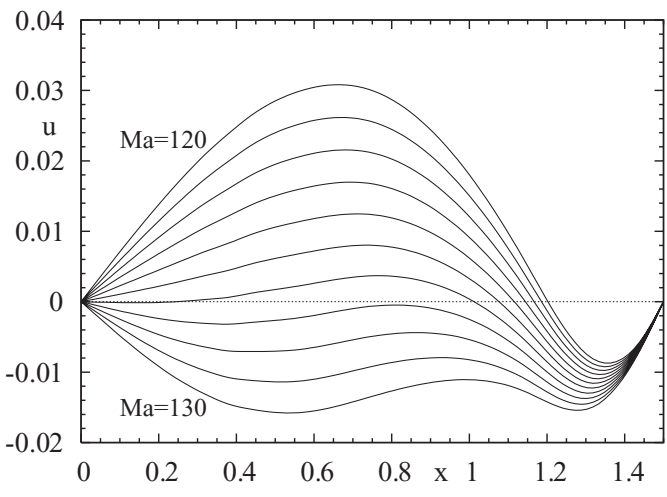

(b)

FIG. 10. Variation with Ma of the radial profiles of the radial surface velocity [plotted as $u(x)$ ] for the critical mode $m=0$ at threshold for $A=1.5$ and $\mathrm{Bi}=1$. The modes are normalized by $w_{\max }$. (a) Profiles for $\mathrm{Ma}=0,50,100,150$, and 200 (solid lines), and for Ma $=120$ and 130 (dashed lines). (b) Profiles for Ma from 120 to 130 (step 1). The dotted line indicates $u=0$. The beginning of flow inversion at the upper surface occurs near the lateral boundary around $\mathrm{Ma}=80$. The complete flow inversion at the upper surface occurs between Ma $=126$ and 127 . 


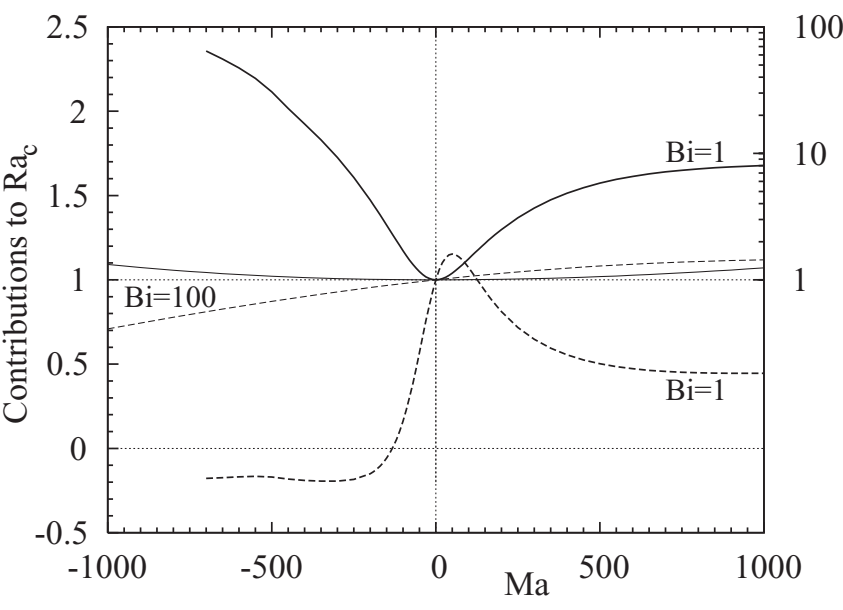

FIG. 11. Energetic contributions to $\mathrm{Ra}_{c}$ given as a function of $\mathrm{Ma}$ for the critical mode $m=0$ at threshold in a cylinder heated from below with a free surface $(A=1.5)$. These contributions given for $\mathrm{Bi}=1$ (thick lines) and $\mathrm{Bi}=100$ (thin lines) are $R_{\text {buoy }}$ (solid lines) and $R_{\text {Mar }}$ (dashed lines), which verify $\mathrm{Ra}_{c} / \mathrm{Ra}_{c, 0}=R_{\text {buoy }} R_{\text {Mar }}$. Note that the variation of $R_{\text {buoy }}$ for $\mathrm{Bi}=1$ (thick solid line) is plotted in the logarithmic scale given on the right of the plot. Dotted lines represent the constant values 1 and 0 and $\mathrm{Ma}=0$.

stronger for $\mathrm{Bi}=1$. This occurs through the decrease of the energy generated by buoyancy $E_{\text {buoy }}^{\prime \prime}$ when surface tension is applied, whatever the sign of Ma is.

For positive Ma, both factors $R_{\text {buoy }}$ and $R_{\text {Mar }}$ increase with $\mathrm{Ma}$ for $\mathrm{Bi}=100$. This indicates that both surface-tension effects and modifications of the buoyancy energy term $E_{\text {buoy }}^{\prime \prime}$ contribute to the increase of $\mathrm{Ra}_{c}$ in this case. For $\mathrm{Bi}=1$, in contrast, the factors $R_{\text {buoy }}$ and $R_{\text {Mar }}$ are together above 1 only for small Ma. For Ma $>126, R_{\text {Mar }}$ becomes less than 1 , indicating a destabilizing influence of surface tension, but it is the strong increase of $R_{\text {buoy }}$ (plotted with regard to the logarithmic scale on the right of the frame in Fig. 11) that will allow the increase of $\mathrm{Ra}_{c}$. This increase of $R_{\text {buoy }}$ corresponds to a strong decrease of the buoyancy energy term $E_{\text {buoy }}^{\prime \prime}$, which, we expect, is due to the constraint imposed on the main toroidal roll by the counter-rotating toroidal cell, which appeared in the upper part of the cavity.

For negative Ma, the Marangoni effect is destabilizing $\left(R_{\mathrm{Mar}}<1\right)$ and the decrease of $R_{\mathrm{Mar}}$ with increasing $|\mathrm{Ma}|$ dominates the increase of $R_{\text {buoy }}$ to induce the decrease of $\mathrm{Ra}_{c}$. For $\mathrm{Bi}=1$, however, $R_{\text {Mar }}$ reaches zero at $\mathrm{Ma}_{c}$ when Marangoni energy equilibrates dissipation and then becomes negative as well as $\mathrm{Ra}_{c}$. Finally, for $\mathrm{Ma}<\mathrm{Ma}_{c}$, the increase of $\left|\mathrm{Ra}_{c}\right|$ is mainly due to the strong increase of $R_{\text {buoy }}$ already mentioned.

\section{B. Nonlinear development of the convection}

To depict the nonlinear development of the convection, we will first present bifurcation diagrams for selected cases and then extend our results by following the paths of the main secondary bifurcation points as a function of $\mathrm{Bi}$ and Ma. Many of the features of the diagrams are dictated by the symmetries of the problem. The axisymmetric flow is triggered by an $m=0$ mode at a transcritical bifurcation point, and two different solution branches are obtained. The asymmetric flows triggered, for example, by the $m=1$ or 2 modes appear at a circular pitchfork bifurcation, and a circle of equivalent solutions defined to within a rotation are obtained. The variable plotted along the ordinate of our bifurcation diagrams as a function of $\mathrm{Ra}$ is the vertical velocity at the center of the cavity $w_{0}$. With this choice, the equivalent solutions generated at a circular pitchfork bifurcation appear as a single curve, whereas the two different solutions generated at a transcritical bifurcation are clearly distinct. In the bifurcation diagrams, solid circles are used to indicate primary and secondary steady bifurcations, while open circles indicate (secondary) Hopf bifurcations. We use solid (dashed) lines to indicate linearly stable (unstable) steady solutions. We also include snapshots of the flow showing the vertical velocity $w$ at midheight in the cavity, with dark (light) shading indicating $w<0(w>0)$. For the solutions that are defined to within a rotation, we have chosen to plot flow structures oriented along the $x$ and $y$ axes.

\section{Bifurcation diagrams}

We have computed bifurcation diagrams in situations without Marangoni effect $(\mathrm{Ma}=0)$ for an aspect ratio $A=$ 1.5, where the first bifurcation produces axisymmetric states. Two values of $\mathrm{Bi}$ have been chosen: $\mathrm{Bi}=100$ and 1 .

Convection for large Biot numbers. The global view of the bifurcation diagram for $\mathrm{Bi}=100$ is presented in Fig. 12, and the first three primary modes at threshold are shown in Fig. 13. The conduction state is stable up to $\operatorname{Ra}_{P_{0}}=1213$, where a transcritical bifurcation to $m=0$ states occurs. At that

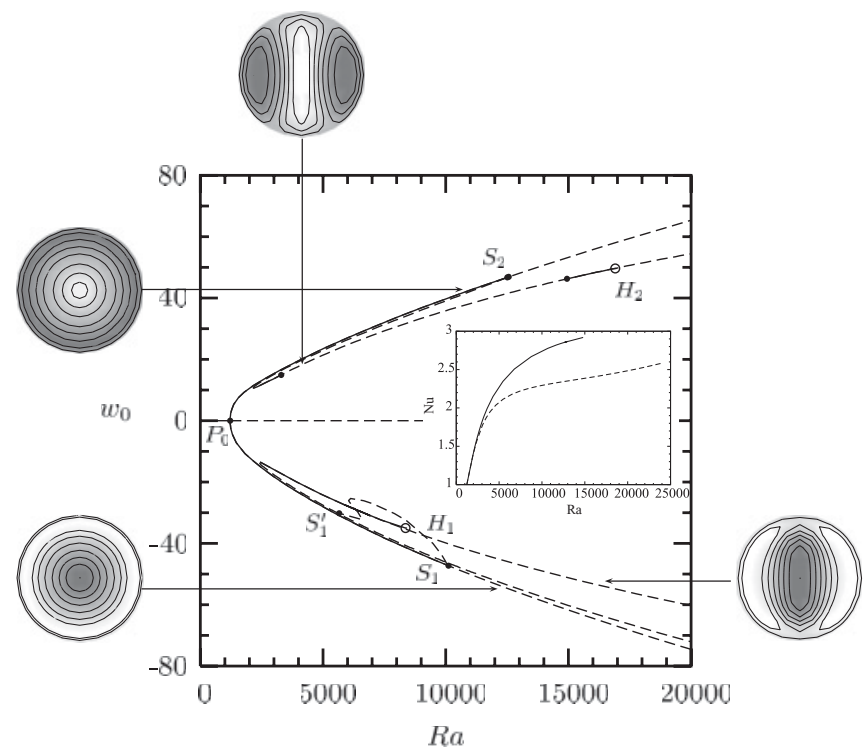

FIG. 12. Bifurcation diagram for a cavity with $A=1.5$ and for $\mathrm{Bi}=100, \mathrm{Ma}=0$, and $\operatorname{Pr}=1$. The bifurcation at $P_{0}$ is transcritical and generates two distinct supercritical (downflow at the center) and subcritical (upflow at the center) axisymmetric branches. The variation with $\mathrm{Ra}$ of the Nusselt number $\mathrm{Nu}$ for these axisymmetric branches is given in the inset. $\mathrm{Nu}$ increases more strongly for the supercritical branch (solid curve) than for the subcritical branch (dashed curve). The flow structure is shown by plots of vertical velocity contours at midheight in the cavity. 


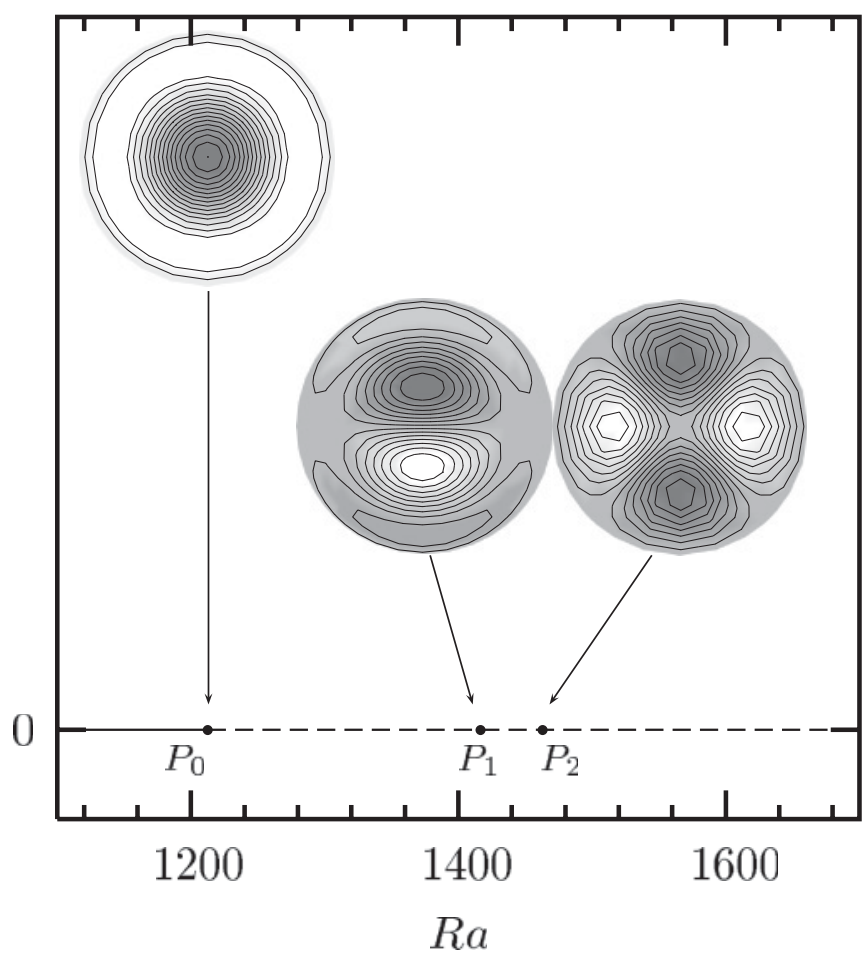

FIG. 13. Vertical velocity contours at midheight in the cavity for the first three critical modes. The bifurcation induced at $P_{0}$ is transcritical, whereas those induced at $P_{1}$ and $P_{2}$ are supercritical circular pitchforks. Only the bifurcation at $P_{0}$ generates stable solutions $(A=1.5, \mathrm{Bi}=100, \mathrm{Ma}=0, \mathrm{Pr}=1)$.

point, two branches of axisymmetric solutions are produced: the supercritical branch corresponds to flows in which the fluid rises toward the free surface along the lateral wall of the cylinder, while the subcritical branch corresponds to flows in which the fluid rises toward the free surface in the center, along the vertical axis. At the onset, the supercritical solutions are stable and the subcritical solutions are unstable. The subcritical branch, however, quickly undergoes a saddle-node bifurcation where it is stabilized. The range of hysteresis between the transcritical and saddle-node bifurcations is too small to be visible on the figure so that the subcritical branch directly appears as stable. This hysteresis will remain very small in any of the bifurcation diagrams we will present, and we shall not refer to it in discussing these diagrams. The evolution of the Nusselt number $\mathrm{Nu}$ as a function of Ra for these two branches of axisymmetric solutions is shown as the inset in Fig. 12. We clearly see that the two branches are distinct branches with different evolutions. The supercritical solutions are found to have a larger Nusselt number than the subcritical solutions. The second primary bifurcation occurs at $\operatorname{Ra}_{P_{1}}=$ 1417 and is a supercritical steady circular pitchfork bifurcation (double critical eigenvalue), which produces a branch of $m=1$ solutions. As can be seen in Fig. 13, the critical $m=1$ mode is a three-roll mode. These solutions inherit the instability of the conduction state in $\mathrm{Ra}_{P_{0}}<\mathrm{Ra}<\mathrm{Ra}_{P_{1}}$ and, hence, are one time unstable at onset (modulo a zero eigenvalue associated with spatial rotations of the pattern). The third primary bifurcation occurs at $\operatorname{Ra}_{P_{2}}=1463$ and is also a supercritical steady circular pitchfork bifurcation, this time producing a branch of

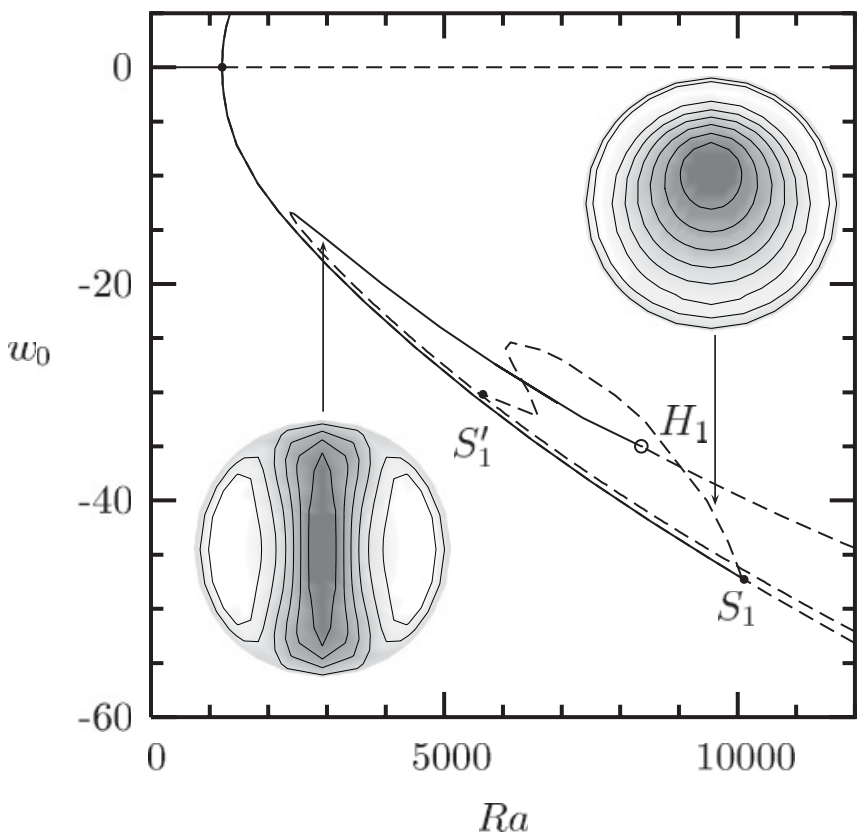

FIG. 14. Zoom of the supercritical part of the axisymmetric branch for a cavity with $A=1.5$ and for $\mathrm{Bi}=100, \mathrm{Ma}=0$, and $\operatorname{Pr}=1$.

$m=2$ solutions, which inherits the three unstable eigenvalues of the conduction state. Both $m=2$ and 1 branches have been found to remain unstable, and we will not discuss them further.

The nonlinear evolution of the axisymmetric branches is rather complex. The supercritical branch (zoomed in Fig. 14) remains stable up to $\mathrm{Ra}_{S_{1}}=10112$, where a circular pitchfork bifurcation associated with $m=1$ perturbations occurs. The figure shows that this bifurcation is subcritical, and the resulting branch of $m=0 / 1$ solutions (with only one vertical plane of symmetry) is therefore one time unstable. This branch progresses toward $\mathrm{Ra}<\mathrm{Ra}_{S_{1}}$ until it undergoes a saddle-node bifurcation at $\mathrm{Ra}=6134$ and reverses direction. At this saddle-node bifurcation, a new unstable eigenvalue appears and the branch therefore remains unstable. The flow structure on this $m=0 / 1$ branch gradually changes to become almost symmetric with respect to a second plane (perpendicular to the first symmetry plane), and the branch eventually connects at $\mathrm{Ra}_{S_{1}^{\prime}}=5782$ with a $m=0 / 2$ solution branch. This new branch, which is unstable at $\mathrm{Ra}_{S_{1}^{\prime}}$, remains unstable for increasing $\mathrm{Ra}$, but acquires stability for decreasing $\mathrm{Ra}$ at a saddle-node bifurcation at $\mathrm{Ra}=2382$. This new solution, which has two perpendicular vertical planes of symmetry, is stable from $\mathrm{Ra}=2382$ to $\mathrm{Ra}_{H_{1}}=8325$, where a Hopf bifurcation is found.

Except for the very small interval between $P_{0}$ and its accompanying saddle-node bifurcation, the subcritical branch, zoomed in Fig. 15, is stable up to $\mathrm{Ra}_{S_{2}}=12513$, where a circular pitchfork bifurcation associated now with $m=2$ perturbations occurs. The bifurcation is here also subcritical, and the resulting branch of $m=0 / 2$ solutions (with two perpendicular vertical planes of symmetry) is therefore one time unstable. This branch strongly progresses leftward, reaching relatively small values of $\mathrm{Ra}$, until it undergoes a 


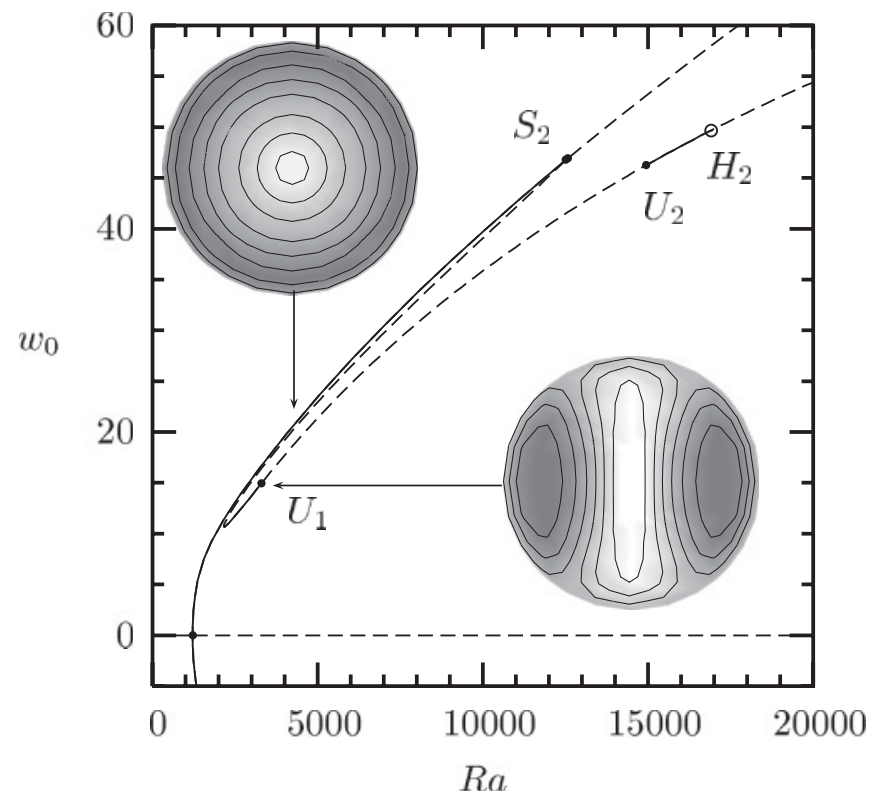

FIG. 15. Zoom of the subcritical part of the axisymmetric branch for a cavity with $A=1.5$ and for $\mathrm{Bi}=100, \mathrm{Ma}=0$, and $\mathrm{Pr}=1$.

saddle-node bifurcation $(\mathrm{Ra}=2154)$ at which it is stabilized. The branch, however, is quickly destabilized at $\mathrm{Ra}_{U_{1}}=3293$ and becomes stable again only beyond $\mathrm{Ra}_{U_{2}}=14948$ before a new destabilization by a Hopf bifurcation at $\mathrm{Ra}_{H_{2}}=16908$. The critical perturbation, which destabilizes the branch in the range $\mathrm{Ra}_{U_{1}}<\mathrm{Ra}<\mathrm{Ra}_{U_{2}}$, is an $m=2$ mode that is oriented at $45^{\circ}$ with respect to the $m=2$ mode at $S_{2}$. This perturbation breaks both symmetries of the solution with respect to vertical planes, but keeps the symmetry with respect to the axis of the cylinder. The evolution with $\mathrm{Ra}$ of the growth rate of the perturbation is presented in Fig. 16. We see that the

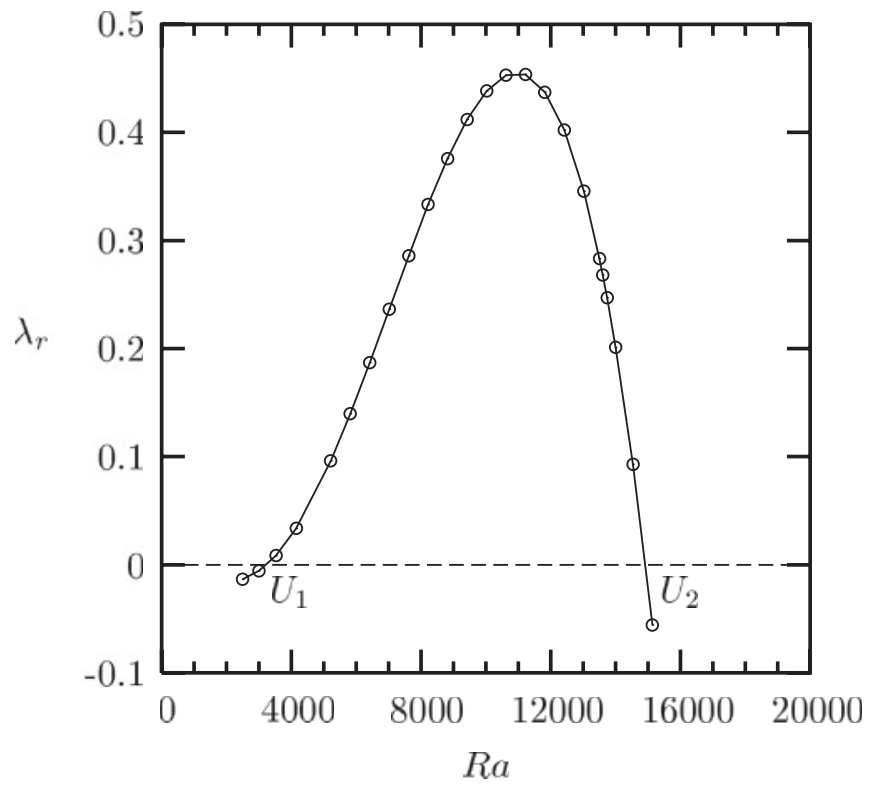

FIG. 16. Growth rate of the critical perturbation that destabilizes and restabilizes the $m=0 / 2$ branch at $U_{1}$ and $U_{2}$, respectively, in the bifurcation diagram of Fig. 15. The parameters are $A=1.5$, $\mathrm{Bi}=100, \mathrm{Ma}=0, \operatorname{Pr}=1$.

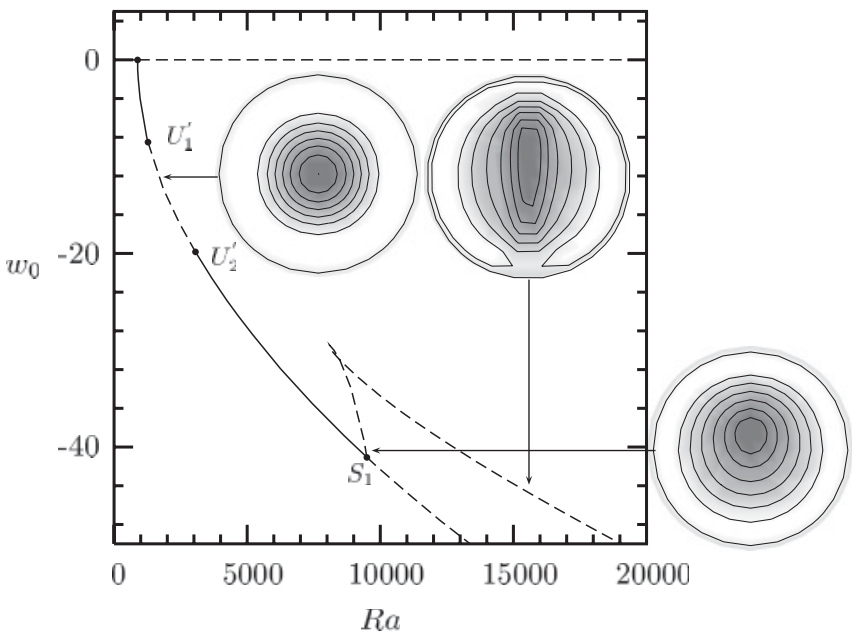

FIG. 17. Bifurcation diagram giving the supercritical part of the axisymmetric branch for a cavity with $A=1.5$ and for $\mathrm{Bi}=1, \mathrm{Ma}=$ 0 , and $\operatorname{Pr}=1$.

growth rate, which becomes positive at $\mathrm{Ra}_{U_{1}}$, first increases with increasing $\mathrm{Ra}$ up to a maximum reached at $\mathrm{Ra} \approx 10900$, and then decreases until it eventually becomes negative at $\operatorname{Ra}_{U_{2}}$. Thus, it is the same $m=2$ mode that destabilizes and restabilizes the $m=0 / 2$ solution branch. Despite multiple attempts, we were not able to get the solution branches that bifurcate at $\mathrm{Ra}_{U_{1}}$ and $\mathrm{Ra}_{U_{2}}$.

Convection for small Biot numbers. We now turn to a discussion of the results obtained for $\mathrm{Bi}=1$. The bifurcation diagram shown in Figs. 17 and 18 has similar features with the diagram obtained for $\mathrm{Bi}=100$, but it also presents clear differences. The primary bifurcations occur in the same order and give successive transitions to $m=0,2$, and 1 states. The first bifurcation at $P_{0}\left(\mathrm{Ra}_{P_{0}}=885\right)$ is transcritical and produces a stable supercritical $m=0$ branch of flows with

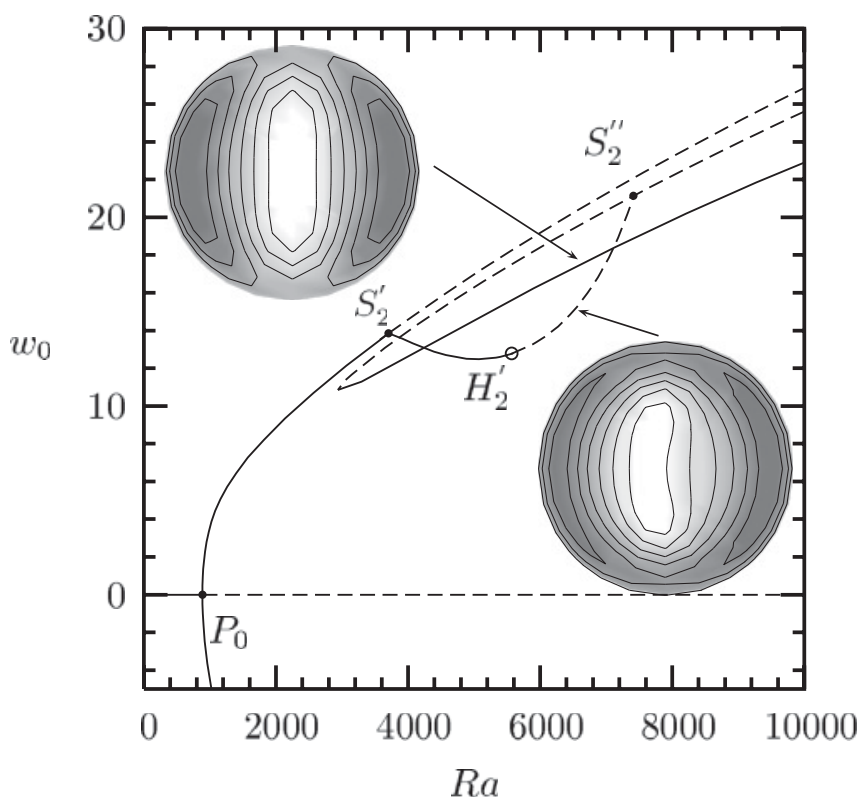

FIG. 18. Bifurcation diagram giving the subcritical part of the axisymmetric branch for a cavity with $A=1.5$ and for $\mathrm{Bi}=1, \mathrm{Ma}=$ 0 , and $\operatorname{Pr}=1$. 
fluid descending in the center and a stable (beyond the very small hysteresis zone) subcritical $m=0$ branch of flows with ascending fluid in the center. The branches of $m=2$ and 1 states, which bifurcate from the conduction state at supercritical circular pitchfork bifurcations $\left(\mathrm{Ra}_{P_{2}}=1023\right.$, $\left.\mathrm{Ra}_{P_{1}}=1115\right)$, remain unstable at larger values of $\mathrm{Ra}$ as for $\mathrm{Bi}=100$ and are not shown on the diagram.

Concerning the nonlinear evolution of the $m=0$ branches, Fig. 17 shows that the supercritical branch is, as for $\mathrm{Bi}=$ 100 , eventually destabilized by $m=1$ perturbations at a circular pitchfork bifurcation. This bifurcation, which occurs at $\mathrm{Ra}_{S_{1}}=9465$, is subcritical, and the resulting branch of $m=0 / 1$ solutions is therefore one time unstable. This branch progresses toward $\mathrm{Ra}<\mathrm{Ra}_{S_{1}}$ until it undergoes a saddle-node bifurcation at $\mathrm{Ra}=8028$ and reverses direction. The branch, one time unstable near $S_{1}$, quickly becomes two times unstable and then undergoes a Hopf bifurcation. One steady eigenvalue is stabilized at the saddle node, but the branch will remain unstable as $\mathrm{Ra}$ is further increased, and will not connect with an $m=0 / 2$ solution branch, in contrast with the results obtained for $\mathrm{Bi}=100$. Note also the local destabilization of the axisymmetric branch between the two bifurcation points at $\mathrm{Ra}_{U_{1}^{\prime}}=1335$ and $\mathrm{Ra}_{U_{2}^{\prime}}=3053$. It is the same $m=2$ mode that is responsible for the successive destabilization and restabilization of the solution. The new branches produced at $U_{1}^{\prime}$ and $U_{2}^{\prime}$ have not been found.

The nonlinear evolution of the subcritical branch, presented in Fig. 18, is strongly modified compared to the case at $\mathrm{Bi}=$ 100. The subcritical branch remains stable up to $\mathrm{Ra}_{s_{2}^{\prime}}=3706$, where a circular pitchfork bifurcation associated now with $m=1$ perturbations occurs. The bifurcation is supercritical, and the resulting branch of $m=0 / 1$ solutions is therefore stable. This branch will remain stable up to a Hopf bifurcation point at $\mathrm{Ra}_{H_{2}^{\prime}}=5567$ and will then terminate on a branch of $m=0 / 2$ states at $\operatorname{Ra}_{S_{2}^{\prime \prime}}=7417$. The evolution with $\mathrm{Ra}$ of the complex unstable eigenvalues created at the Hopf bifurcation is shown in Fig. 19. We see that they collide on the positive real axis at $\mathrm{Ra} \approx 6800$. Another Hopf bifurcation occurs close above $\mathrm{Ra}_{H_{2}^{\prime}}$. The corresponding complex eigenvalues very quickly collide on the positive real axis (at $\mathrm{Ra} \approx 5885$ ), and one of the steady eigenvalues is further restabilized. The $m=0 / 1$ branch is then three times unstable close to $S_{2}^{\prime \prime}$. The new branch of $m=0 / 2$ states inherits the instability of the $m=0 / 1$ branch and, hence, is three times unstable close above $S_{1}^{\prime \prime}$. Below $S_{1}^{\prime \prime}$, it becomes two times unstable and then once unstable at a further steady bifurcation $(\mathrm{Ra}=4259)$ before it turns around at a saddle-node bifurcation $(\mathrm{Ra}=2937)$ and acquires stability. The branch will remain stable at least up to $\mathrm{Ra}=20000$. This $m=0 / 2$ branch looks quite similar to that obtained for $\mathrm{Bi}=100$. The subcritical circular pitchfork bifurcation point $S_{2}$ at which it is created, however, has moved to much larger values of $\mathrm{Ra}$ (outside the range of $\mathrm{Ra}$ values considered in the study) and is no more the first bifurcation point on the subcritical $m=0$ branch.

In the case $\mathrm{Bi}=1$ for a cylinder of aspect ratio $A=1.5$, we can summarize the sequence of stable steady solutions that might be observable in an experiment. These solutions correspond to several different flows: axisymmetric steady flows with upflow or downflow in the center, $m=0 / 1$ and $m=0 / 2$ steady flows with upflow in the center. All these different patterns are observable in appropriate ranges of Ra: axisymmetric flows at the onset of convection from $P_{0}$ to $U_{1}^{\prime}$ and from $U_{2}^{\prime}$ to $S_{1}$ (downflow in the center) or from $P_{0}$ to $S_{2}^{\prime}$ (upflow in the center), $m=0 / 1$ steady flows from $S_{2}^{\prime}$ to $H_{2}^{\prime}$, and $m=0 / 2$ steady flows above the saddle node where they acquire stability. Note that the sequence of observable solutions for increasing and then decreasing Ra leads to interesting hysteresis phenomena.

\section{Paths of the secondary bifurcation points}

In order to make a connection between the results obtained for $\mathrm{Bi}=1$ and 100, we have followed the main characteristic points of the bifurcation diagrams as a function of the Biot number. This will allow us to see how the existence range of the observable steady solutions evolve as $\mathrm{Bi}$ is changed.

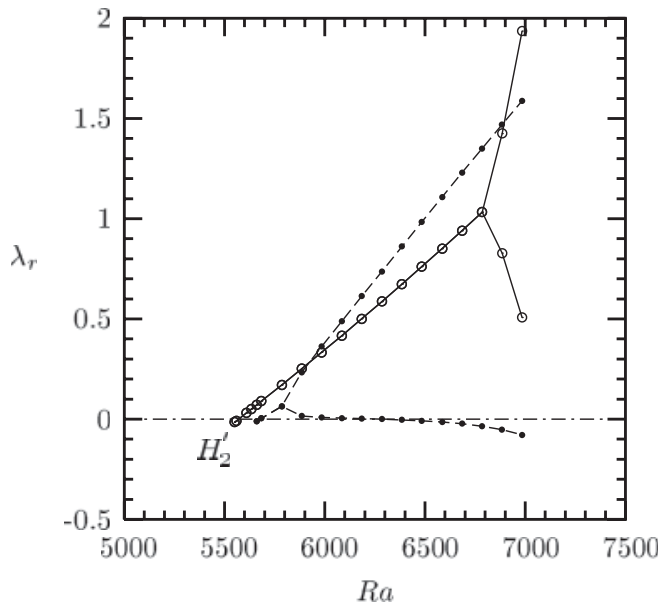

(a)

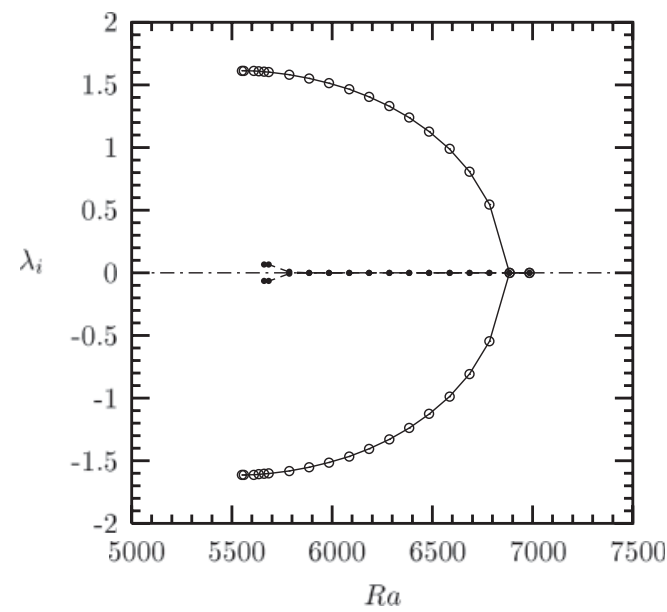

(b)

FIG. 19. Variation of the dominant eigenvalues along the $m=0 / 1$ branch beyond the $\mathrm{Ra}_{H_{2}^{\prime}}$ threshold. The real part of the eigenvalues is shown in (a) and the imaginary part in (b). Each oscillatory eigenvalue splits into two steady eigenvalues. 


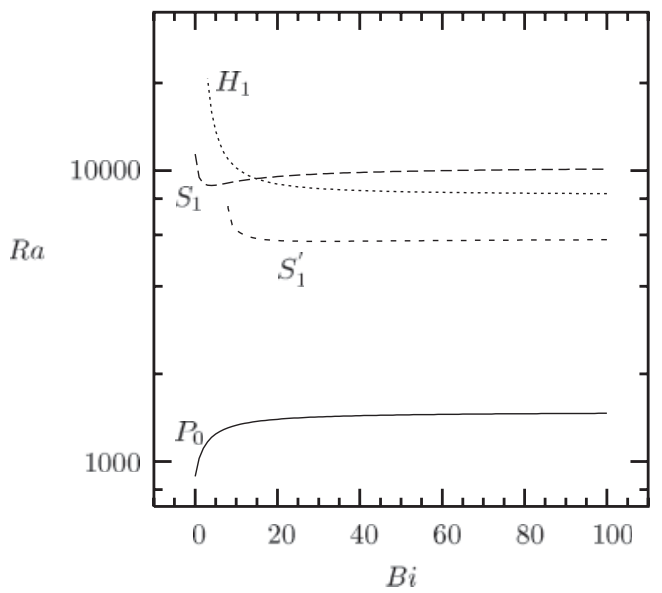

(a)

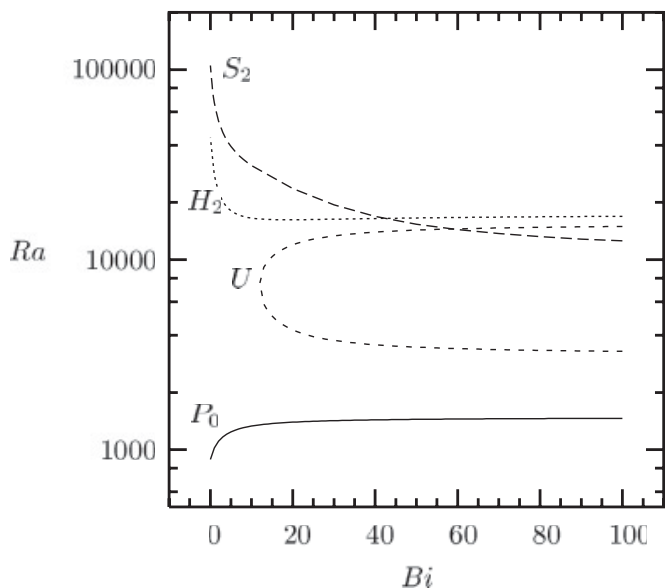

(b)

FIG. 20. Paths for decreasing Bi of the secondary thresholds obtained from the supercritical (a) and subcritical (b) axisymmetric branches of the bifurcation diagram calculated for $A=1.5, \mathrm{Bi}=100, \mathrm{Ma}=0, \mathrm{Pr}=1$. The primary threshold $\mathrm{Ra}_{P_{0}}$ is given for comparison.

We first consider the bifurcation points on the supercritical axisymmetric branch at $\mathrm{Bi}=100$. Four points are followed for decreasing $\mathrm{Bi}$ in Fig. 20(a): the primary threshold $\mathrm{Ra}_{P_{0}}$, the limit points of the $m=0 / 1$ branches $\operatorname{Ra}_{S_{1}}$ and $\mathrm{Ra}_{S_{1}^{\prime}}$ and the Hopf bifurcation point $\mathrm{Ra}_{H_{1}}$, which destabilizes the $m=0 / 2$ branch. We see that, down to $\mathrm{Bi}=20$, the variation of these different points is very weak, the Ra values changing by less than $8 \%$ compared to the values at $\mathrm{Bi}=100$. In this range of $\mathrm{Bi}$, the nonlinear evolution of the convection is globally unchanged, both in terms of flow structure and values of the thresholds. For weaker values of $\mathrm{Bi}$, in contrast, we find a significant variation of these bifurcation points. Below $\mathrm{Bi}=20, \mathrm{Ra}_{S_{1}}$ first slightly decreases, then increases for small values of $\mathrm{Bi}(\mathrm{Bi} \leqslant 3)$ and eventually reaches its maximum value $\mathrm{Ra}_{S_{1}}=11392$ for $\mathrm{Bi}=0$. As the primary threshold $\mathrm{Ra}_{P_{0}}$ is found to decrease below $\mathrm{Bi}=20$, we then get an increase of the $\mathrm{Ra}$ range where the supercritical axisymmetric branch can be experimentally observed. The two other secondary thresholds $\mathrm{Ra}_{S_{1}^{\prime}}$ and $\mathrm{Ra}_{H_{1}}$ gradually increase as $\mathrm{Bi}$ is decreased below $\mathrm{Bi}=20$. These thresholds, however, were not obtained down to $\mathrm{Bi}=0$, but only down to $\mathrm{Bi}=8$ and 3, respectively, where the maximum values reached are $\mathrm{Ra}_{S_{1}^{\prime}}=7432$ and $\mathrm{Ra}_{H_{1}}=20692$. A zoomed view of the small Bi range is shown in Fig. 21(a). In this figure, we can see the evolution of the additional points $U_{1}^{\prime}$ and $U_{2}^{\prime}$ found for $\mathrm{Bi}=1$. These points get closer as $\mathrm{Bi}$ is increased and quickly collide and disappear around $\mathrm{Bi}=2.5$. The unstable $\mathrm{Ra}$ range between $\mathrm{Ra}_{U_{1}^{\prime}}$ and $\mathrm{Ra}_{U_{2}^{\prime}}$ thus does not exist any more beyond this Bi value.

We now consider the subcritical axisymmetric branch. The characteristic bifurcation points for $\mathrm{Bi}=100$ and 1 are different for this branch, which means that a transition is expected as $\mathrm{Bi}$ is changed from 100 to 1 . The evolution with $\mathrm{Bi}$ of the thresholds $S_{2}, H_{2}, U_{1}$, and $U_{2}$ found for $\mathrm{Bi}=100$ is shown in Fig. 20(b). The $S_{2}$ threshold, at which the subcritical axisymmetric branch is destabilized by an $m=2$ perturbation, experiences a regularly amplified increase as $\mathrm{Bi}$ is decreased and eventually reaches its maximum value $\mathrm{Ra}_{S_{2}}=105198$

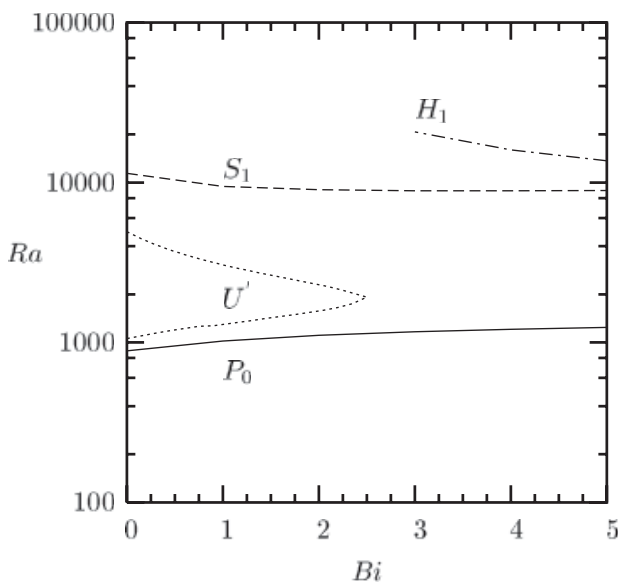

(a)

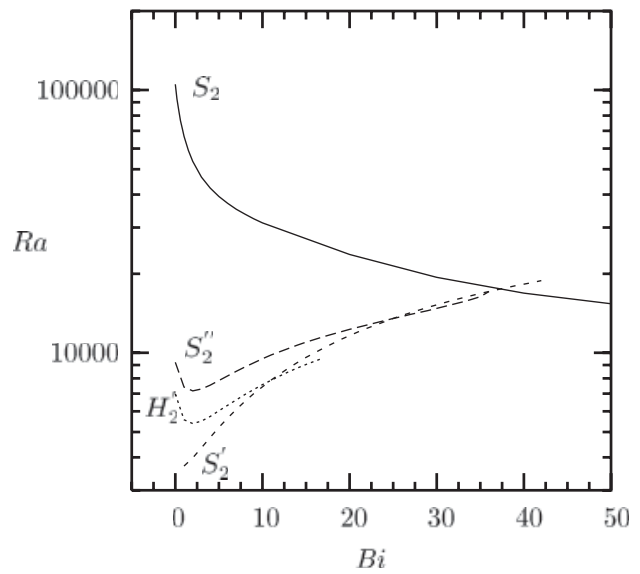

(b)

FIG. 21. Paths for increasing Bi of the secondary thresholds obtained from the supercritical (a) and subcritical (b) axisymmetric branches of the bifurcation diagram calculated for $A=1.5, \mathrm{Bi}=1, \mathrm{Ma}=0, \mathrm{Pr}=1$. The primary threshold $\mathrm{Ra}_{P_{0}}$ is given for comparison. 
at $\mathrm{Bi}=0$. On the $m=0 / 2$ branch, initiated subcritically at $S_{2}$ and stabilized at a saddle-node point, the Ra range between $\mathrm{Ra}_{U_{1}}$ and $\mathrm{Ra}_{U_{2}}$, where destabilization occurs, slowly decreases as $\mathrm{Bi}$ is decreased. This decrease is amplified below $\mathrm{Bi}=40$ and the destabilization zone eventually disappears when the two points collide at $\mathrm{Ra}_{U}=7550$ for $\mathrm{Bi} \approx 12$. This corresponds to a modification of the amplification rate of the perturbation. This amplification rate (Fig. 16) has a maximum between $\mathrm{Ra}_{U_{1}}$ and $\mathrm{Ra}_{U_{2}}$, which is positive for large values of $\mathrm{Bi}$, decreases as $\mathrm{Bi}$ is decreased, and eventually becomes negative for $\mathrm{Bi}<12$. Finally, the Hopf bifurcation point $H_{2}$, at which the $m=0 / 2$ branch is definitively destabilized, has an evolution with Bi qualitatively similar as the point $H_{1}$ found in the supercritical part of the diagram. Down to $\mathrm{Bi}=10$, its variation is very weak and below this value, there is an increase up to the value $\mathrm{Ra}_{H_{2}}=69788$ reached for $\mathrm{Bi}=0$.

Except for $U_{1}$ and $U_{2}$, the main bifurcation points $S_{2}$ and $\mathrm{H}_{2}$ have not disappeared and are still present for $\mathrm{Bi}=0$. The corresponding Rayleigh numbers $\mathrm{Ra}_{S_{2}}$ and $\mathrm{Ra}_{H_{2}}$, however, have strongly increased, so that these bifurcations are not the first ones to appear in the diagram. As an example, on the subcritical axisymmetric branch, the bifurcation at $\mathrm{Ra}_{S_{2}}$ (leading to the $m=0 / 2$ branch) is now preceded by the bifurcation leading to the $m=0 / 1$ branch at $\mathrm{Ra}_{S_{2}^{\prime}}$. The saddle node on the $m=0 / 2$ branch, in contrast, has not much evolved, which indicates that the subcriticality of this branch becomes really strong for the weak values of $\mathrm{Bi}$. The diagram obtained for $\mathrm{Bi}=1$ has shown which bifurcation points first appear for small $\mathrm{Bi}$ values. It is then interesting to follow these points $\mathrm{Ra}_{S_{2}^{\prime}}, \mathrm{Ra}_{S_{2}^{\prime \prime}}$, and $\mathrm{Ra}_{H_{2}^{\prime}}$ as $\mathrm{Bi}$ is increased. The results are shown in Fig. 21(b).

The variation with increasing $\mathrm{Bi}$ of the bifurcation points $\mathrm{Ra}_{S_{2}^{\prime \prime}}$ and $\mathrm{Ra}_{H_{2}^{\prime}}$ is quite similar and corresponds to an abrupt decrease followed by a regular increase, whereas a monotonous increase is found for $\mathrm{Ra}_{S_{2}^{\prime}}$. The Hopf bifurcation at $\mathrm{Ra}_{H_{2}^{\prime}}$, however, disappears for $\mathrm{Bi} \approx 17$ and the steady bifurcation at $\mathrm{Ra}_{S_{2}^{\prime \prime}}$ also disappears for $\mathrm{Bi} \approx 36$ at the intersection point between the first thresholds on the subcritical axisymmetric branches $\mathrm{Ra}_{S_{2}^{\prime}}$ and $\mathrm{Ra}_{S_{2}}$ [the other intersections in Fig. 21(b) are not real intersection points but merely coincident values of the thresholds for bifurcation points that are not on the same portion of curve]. Some additional calculations have shown that the $m=0 / 1$ branch, which is supercritical for $\mathrm{Bi}=1$, emerges subcritically at $\mathrm{Ra}_{S_{2}^{\prime}}$ for $\mathrm{Bi}=17$ and 40 and changes direction at a saddle-node point.

From these results, it appears that, when the bifurcation points at which the $m=0 / 1$ and $0 / 2$ branches are produced, $\mathrm{Ra}_{S_{2}^{\prime}}$ and $\mathrm{Ra}_{S_{2}}$, respectively, meet for decreasing $\mathrm{Bi}$, a new bifurcation point $\operatorname{Ra}_{S_{2}^{\prime \prime}}$ is produced at which the $m=0 / 1$ branch now connects the $m=0 / 2$ branch. The way the $m=0 / 1$ branch emerges at $\mathrm{Ra}_{S_{2}^{\prime}}$ also changes from subcritical for large values of $\mathrm{Bi}$ to supercritical for small values of $\mathrm{Bi}$. Finally, it can be expected that the Hopf bifurcation point $\mathrm{Ra}_{H_{2}^{\prime}}$ found for small values of $\mathrm{Bi}$ on the $m=0 / 1$ branch disappears by colliding with the saddle node of this branch.

Finally, the influence of the Marangoni number Ma on the secondary bifurcation thresholds $\mathrm{Ra}_{S_{1}}$ and $\mathrm{Ra}_{S_{2}}$ is shown in Fig. 22 for $\mathrm{Bi}=100$. We find an increase of the thresholds

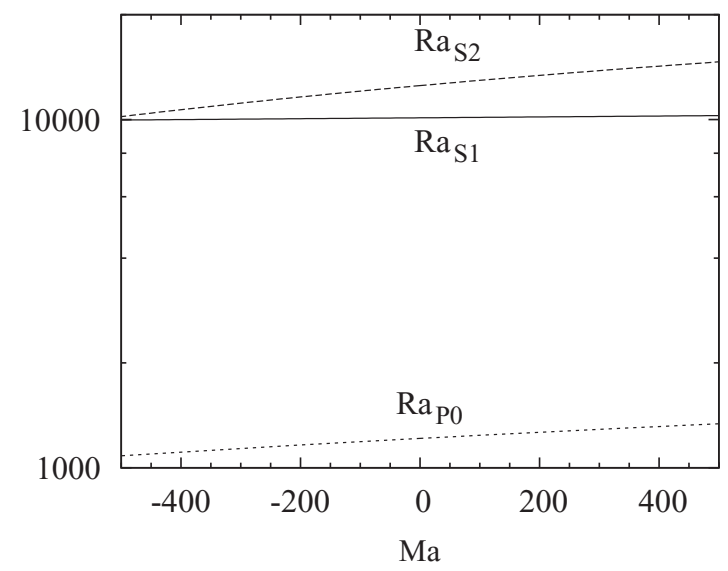

FIG. 22. Variation of the primary threshold $\mathrm{Ra}_{P_{0}}$ and secondary thresholds $\mathrm{Ra}_{S_{1}}$ and $\mathrm{Ra}_{S_{2}}$ as a function of the Marangoni number Ma for $A=1.5, \mathrm{Bi}=100$, and $\operatorname{Pr}=1$.

with $\mathrm{Ma}$, but for this value of $\mathrm{Bi}$, the increase remains moderate for $\mathrm{Ra}_{S_{2}}$ and is rather small for $\mathrm{Ra}_{S_{1}}$.

\section{Temperature at the free surface}

In our situation, the temperature is only prescribed at the bottom plate and we have a heat exchange condition at the upper free surface. In dimensionless form, this gives a temperature fixed to 1 at the bottom plate, whereas its value at the free surface is zero in the diffusive regime but will change with the onset of convection. To see how the temperature at the free surface is modified by the convection, we analyze the axisymmetric solutions at $S_{1}$ and $S_{2}$ when $\mathrm{Bi}$ is changed.

We first give the variation with $\mathrm{Bi}$ of the temperature difference $\Delta T$ between the bottom and the upper surfaces along the cylinder axis for the two solutions in Fig. 23. (Note that $\Delta T$ would be 1 in the diffusive regime.) We see that, for $\mathrm{Bi}=100, \Delta T$ is slightly larger than 1 for the solution $S_{1}$ with downflow at the center, whereas it is smaller than 1 for the

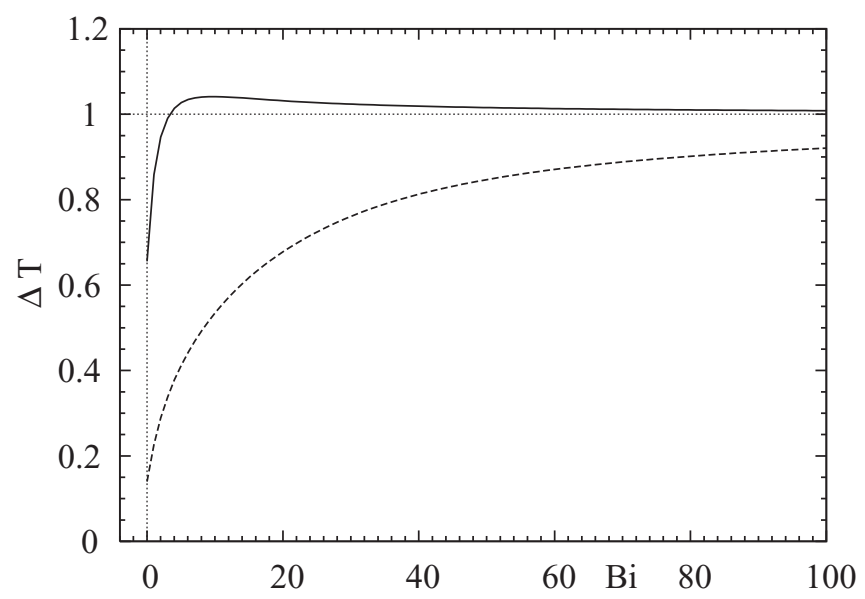

FIG. 23. Variation with $\mathrm{Bi}$ of the temperature difference $\Delta T$ between the bottom and the upper surfaces along the cylinder axis for the $m=0$ solution at the secondary thresholds $\mathrm{Ra}_{S_{1}}$ (solid line) and $\mathrm{Ra}_{S_{2}}$ (dashed line) for $A=1.5$ and $\mathrm{Ma}=0$. The dotted lines indicate $\mathrm{Bi}=0$ and $\Delta T=1$. 
solution $S_{2}$ with upflow at the center. In fact, upflow is expected to carry hot fluid from the bottom to the free surface and then to decrease $\Delta T$ compared to the diffusive regime. When $\mathrm{Bi}$ is decreased, two effects are involved in the change of $\Delta T$ : first, the temperature is less constrained at the free surface for small $\mathrm{Bi}$, which allows easier temperature modifications; then, the Ra values at $S_{1}$ and $S_{2}$ are changed, which modifies the convection. For the solution $S_{1}, \Delta T$ is principally changed for small values of $\mathrm{Bi}$ where the decrease down to 0.656 for $\mathrm{Bi}=0$ is due to both the decrease of $\mathrm{Bi}$ and the increase of $\mathrm{Ra}$. For the solution $S_{2}$, the decrease of $\Delta T$ as $\mathrm{Bi}$ is decreased is at large $\mathrm{Bi}$ principally due to the increase of $\mathrm{Ra}$, whereas for small $\mathrm{Bi}$, both effects are effective. A small value, $\Delta T=0.140$, is reached for $\mathrm{Bi}=0$ in this case.

The temperature profile along the free surface is then given in Fig. 24 for the same solutions at $S_{1}$ and $S_{2}$ and for different $\mathrm{Bi}$ numbers. We see that the temperature at the free surface is the largest in the zones where the fluid moves up, i.e., close

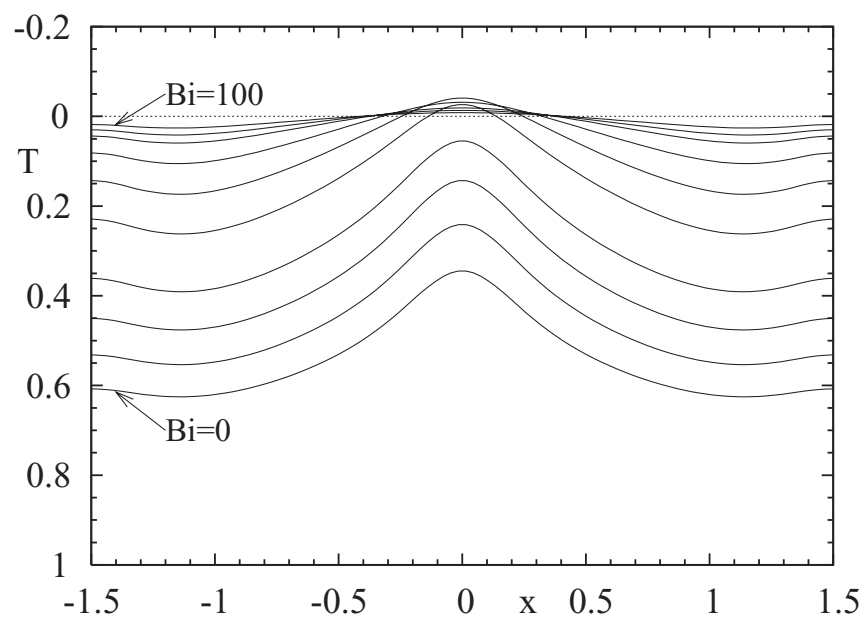

(a)

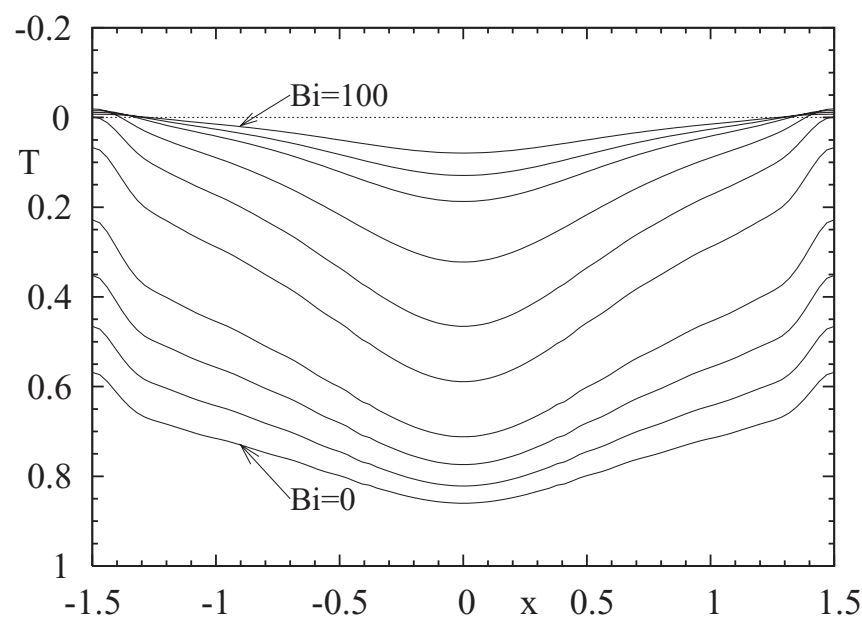

(b)

FIG. 24. Variation with $\mathrm{Bi}$ of the radial profiles of the surface temperature [plotted as $T(x)$ ] for the $m=0$ solution at the secondary thresholds $\mathrm{Ra}_{S_{1}}$ (a) and $\mathrm{Ra}_{S_{2}}$ (b) for $A=1.5$ and $\mathrm{Ma}=0$. The profiles are given for $\mathrm{Bi}=100,60,40,20,10,5,2,1,0.4$, and 0 . The dotted line indicates $T=0$. to the lateral boundaries for the $S_{1}$ solutions and along the cylinder axis for the $S_{2}$ solutions, and the smallest in the zones where the fluid moves down, i.e., along the cylinder axis for the $S_{1}$ solutions and along the lateral boundaries for the $S_{2}$ solutions. For both solutions, we find a global increase of the temperature when $\mathrm{Bi}$ becomes small. This global increase of the temperature is stronger for the $S_{2}$ solutions for which a strong increase of $\mathrm{Ra}$ occurs, but it is also important (if $\mathrm{Bi}$ is sufficiently decreased) for the $S_{1}$ solutions for which Ra is not much changed. Finally, we can see that the increase of the temperature is continuous in the zones with upflow, whereas in the zones with downflow, we find a first slight decrease of the temperature toward slightly negative values before the increase. The minimum temperature in these zones is obtained for $\mathrm{Bi} \approx 10$ for the $S_{1}$ solutions and $\mathrm{Bi} \approx 20$ for the $S_{2}$ solutions.

\section{Energy budgets at the main secondary thresholds}

The kinetic energy budgets at the secondary thresholds $\mathrm{Ra}_{S_{1}}$ and $\mathrm{Ra}_{S_{2}}$ [buoyancy term $E_{\text {buoy }}^{\prime}$, shear term $E_{\text {shear }}^{\prime}$, surface tension term $E_{\mathrm{Mar}}^{\prime}$ equal to 0 as $\mathrm{Ma}=0$, and viscous dissipation term normalized to - 1, as shown in Eq. (10)] were calculated from the flow solution and critical eigenvector. The energy contributions $E_{\text {buoy }}^{\prime}$ and $E_{\text {shear }}^{\prime}$ are shown as a function of Bi for both thresholds in Fig. 25. We see that the instabilities at $S_{1}$ and $S_{2}$ are still dominated by buoyancy effects. The shear energy, however, has a non-negligible contribution. If we compare both cases at $\mathrm{Bi}=100$, where $\mathrm{Ra}_{S_{1}}$ and $\mathrm{Ra}_{S_{2}}$ are not very different, we see that the shear contribution is more important at $S_{1}$, i.e., in the case where an $m=1$ mode is triggered from a solution with downflow at the center, than at $S_{2}$, i.e., in the case where an $m=2$ mode is triggered from a solution with upflow at the center. When $\mathrm{Bi}$ is decreased, in contrast, the shear energy at $S_{2}$ becomes more important because of the strong increase of $\mathrm{Ra}_{S_{2}}$.

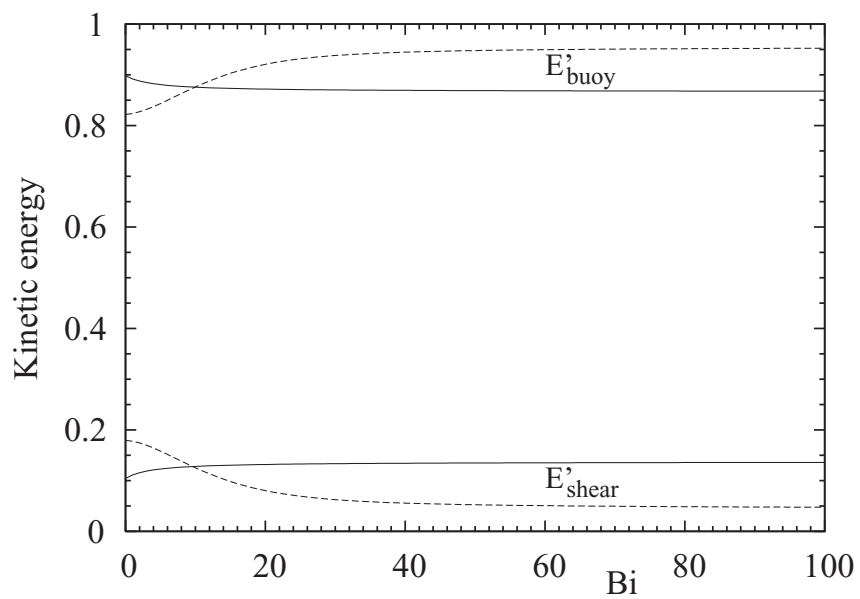

FIG. 25. Kinetic energy budget associated with the perturbations at the secondary bifurcation points $S_{1}$ (solid lines) and $S_{2}$ (dashed lines) for a cylinder heated from below with a free surface $(A=1.5)$. The contributions $E_{\text {buoy }}^{\prime}$ and $E_{\text {shear }}^{\prime}$ are given as a function of the Biot number for $\mathrm{Ma}=0$. 


\section{CONCLUSION}

The development of convection in a cylindrical cavity heated from below and with a free surface at the top has been numerically investigated. The influence of buoyancy and surface tension has been considered together with the heat exchange at the upper free surface.

The onset of convection has been first studied by following the paths of the primary bifurcation points (expressed as a critical Rayleigh number $\mathrm{Ra}_{c}$ ) as a function of the important parameters of the problem, the aspect ratio of the cavity $A$, the Biot number Bi, and the Marangoni number Ma (chosen as stabilizing when positive). These primary thresholds are associated with azimuthal eigenmodes with different symmetry properties. The more dangerous eigenmodes are the first azimuthal modes and, principally, for rather confined cylinders, the one-roll $m=1$ mode and the axisymmetric $m=0$ mode, which are successively dominant as $A$ is increased. Particular attention has been paid to the influence of the Biot and Marangoni numbers. An increase of Ma induces an increase of the critical Rayleigh number $\mathrm{Ra}_{c}$, but this increase, strongly depending on $\mathrm{Bi}$, is the strongest for small $\mathrm{Bi}$ values and very weak for large $\mathrm{Bi}$, a situation where the temperature at the free surface is nearly constant. A positive value $\mathrm{Ma}_{B}$ of the Marangoni number has been detected for which the Biot number has no effect on the primary thresholds: above this value, the thresholds increase as $\mathrm{Bi}$ is decreased, and below, they decrease as $\mathrm{Bi}$ is decreased. Interesting information on the influence of Ma has been found. For a negative value $\mathrm{Ma}_{c}$, the onset of convection occurs at $\mathrm{Ra}=0$ (pure Marangoni threshold), and for $\mathrm{Ma}<\mathrm{Ma}_{c}$, the onset is for negative values of $\mathrm{Ra}_{c}$, i.e., in situations heated from above. For positive values of $\mathrm{Ma}$, the increase of the primary thresholds is first due to a surface-tension force opposing the flow induced by buoyancy. Beyond a given value $\mathrm{Ma}_{I}$ depending on $\mathrm{Bi}$, however, counter-rotating rolls are created near the free surface, and the stabilizing effect is then due to the confinement exerted by these new rolls on the buoyant flow. The progressive change of the surface velocity, which gives rise to these new rolls, has been studied in detail.

The nonlinear evolution of the convection has been studied for a cavity with $A=1.5, \mathrm{Ma}=0$, and different values of $\mathrm{Bi}$. In such a cavity, the onset of convection is to axisymmetric flows, which appear at a transcritical bifurcation point. Two branches of axisymmetric solutions are created, a supercritical branch corresponding to solutions with downflow in the center of the cylinder and a subcritical branch corresponding to solutions with upflow at the center. These solutions are not equivalent: they correspond to different evolutions of the Nusselt number and they are destabilized at different secondary bifurcation points. For $\mathrm{Bi}=100$, it is an $m=1$ mode that destabilizes the solutions on the supercritical branch at $\mathrm{Ra}_{s_{1}}$ and an $m=2$ mode that destabilizes the solutions on the subcritical branch at $\mathrm{Ra}_{S_{2}}$. These bifurcations are strongly subcritical and generate unstable branches. In both cases, however, it is $m=0 / 2$ solutions that are eventually stabilized at saddle-node points. For $\mathrm{Bi}=1$, the results are much changed. For example, stable $m=0 / 1$ solutions can now be obtained on a branch that bifurcates supercritically at $\mathrm{Ra}_{S_{2}^{\prime}}$ from the subcritical axisymmetric branch. The changes that affect the bifurcation diagram when $\mathrm{Bi}$ is decreased from $\mathrm{Bi}=100$ to 1 have been highlighted by following the paths of the main secondary bifurcation points. It is then found that the decrease of $\mathrm{Bi}$ does not affect much $\mathrm{Ra}_{S_{1}}$, but induces a strong increase of $\mathrm{Ra}_{S_{2}}$ which, below $\mathrm{Bi} \approx 36$, gives place to $\mathrm{Ra}_{S_{2}^{\prime}}$ as the first threshold. Concerning the influence of $\mathrm{Ma}$ for $\mathrm{Bi}=100$, it is rather weak for $\mathrm{Ra}_{S_{1}}$, whereas an increase of $\mathrm{Ra}_{S_{2}}$ is found for increasing Ma. Finally, the temperature variations induced at the free surface by the convection have been investigated. The temperature at the free surface, which is zero in the diffusive situation, is modified by convection. For large $\mathrm{Bi}($ as $\mathrm{Bi}>20$ ) and moderate $\mathrm{Ra}($ as $\mathrm{Ra} \approx 10000$ ), the temperature is increased in the zones with upflow (hot fluid is carried from the heated bottom) and decreased in the zones with downflow. For smaller Bi or larger $\mathrm{Ra}$, the temperature is increased along the whole free surface, but more strongly in the zones with upflow. The temperature difference between the bottom and the top, which is 1 in the diffusive situation, can be strongly reduced down to 0.4 when we follow the solution at $\mathrm{Ra}_{S_{1}}$ for decreasing Bi and even down to 0.14 when we follow the solution at $\mathrm{Ra}_{S_{2}}$.

Further studies concerning the influence of a horizontal magnetic field are reported in the companion paper [31].

\section{ACKNOWLEDGMENT}

This work was granted access to the HPC resources of IDRIS under the allocations 2008-021559, 2009-021559, 2010-021559, and 2011-021559 made by GENCI (Grand Equipement National de Calcul Intensif).
[1] H. Bénard, Annal. Chim. Phys. 23, 62 (1901).

[2] E. L. Koschmieder, Bénard Cells and Taylor Vortices (Cambridge University Press, Cambridge, 1993).

[3] Lord Rayleigh, Philos. Mag. 32, 529 (1916).

[4] J. R. A. Pearson, J. Fluid Mech. 4, 489 (1958).

[5] D. A. Nield, J. Fluid Mech. 19, 341 (1964).

[6] E. L. Koschmieder and S. A. Prahl, J. Fluid Mech. 215, 571 (1990).

[7] D. Johnson and R. Narayanan, Phys. Rev. E 54, 3102 (1996).
[8] P. Cerisier, S. Rahal, and H. Azuma, J. Phys.: Conf. Ser. 64, 012004 (2007).

[9] S. Rahal, P. Cerisier, and H. Azuma, Exp. Fluids 43, 547 (2007).

[10] A. Bergeon, D. Henry, H. Ben Hadid, and L. S. Tuckerman, J. Fluid Mech. 375, 143 (1998).

[11] S. Rosenblat, S. Davis, and G. Homsy, J. Fluid Mech. 120, 91 (1982)

[12] S. Rosenblat, G. Homsy, and S. Davis, J. Fluid Mech. 120, 123 (1982). 
[13] P. C. Dauby, G. Lebon, P. Colinet, and J. C. Legros, Q. J. Mech. Appl. Math. 46, 683 (1993).

[14] H. A. Dijkstra, J. Fluid Mech. 243, 73 (1992).

[15] H. A. Dijkstra, Microgravity Sci. Technol. 7, 307 (1995).

[16] H. A. Dijkstra, Microgravity Sci. Technol. 8, 70 (1995).

[17] H. A. Dijkstra, Microgravity Sci. Technol. 8, 155 (1995).

[18] P. C. Dauby and G. Lebon, J. Fluid Mech. 329, 25 (1996).

[19] J. S. Vrentas, R. Narayanan, and S. S. Agrawal, Int. J. Heat Mass Transfer 24, 1513 (1981).

[20] G. S. Charlson and R. L. Sani, Int. J. Heat Mass Transfer 13, 1479 (1970).

[21] C. Wagner, R. Friedrich, and R. Narayanan, Phys. Fluids 6, 1425 (1994).

[22] A. A. Zaman and R. Narayanan, J. Colloid Interface Sci. 179, 151 (1996).
[23] P. C. Dauby, G. Lebon, and E. Bouhy, Phys. Rev. E 56, 520 (1997).

[24] P. Assemat, A. Bergeon, and E. Knobloch, Phys. Fluids 19, 104101 (2007).

[25] R. Touihri, H. Ben Hadid, and D. Henry, Phys. Fluids 11, 2078 (1999).

[26] G. E. Karniadakis, M. Israeli, and S. A. Orszag, J. Comput. Phys. 97, 414 (1990).

[27] C. K. Mamun and L. S. Tuckerman, Phys. Fluids 7, 80 (1995).

[28] D. Henry and H. Ben Hadid, Phys. Rev. E 76, 016314 (2007).

[29] D. R. Kincaid, T. C. Oppe, and W. D. Joubert, Comput. Phys. Commun. 53, 283 (1989).

[30] D. A. Nield, J. Fluid Mech. 29, 545 (1967).

[31] R. Touihri, A. El Gallaf, H. Ben Hadid, and D. Henry, Phys. Rev. E 84, 056303 (2011). 